\title{
Improved hydrogeophysical characterization using joint inversion of cross-hole electrical resistance and ground-penetrating radar traveltime data
}

\author{
Niklas Linde, ${ }^{1,2,3}$ Andrew Binley, ${ }^{4}$ Ari Tryggvason, ${ }^{1}$ Laust B. Pedersen, ${ }^{1}$ and André Revil ${ }^{2}$ \\ Received 27 April 2006; revised 24 July 2006; accepted 16 August 2006; published 12 December 2006.
}

[1] Appropriate regularizations of geophysical inverse problems and joint inversion of different data types improve geophysical models and increase their usefulness in hydrogeological studies. We have developed an efficient method to calculate stochastic regularization operators for given geostatistical models. The method, which combines circulant embedding and the diagonalization theorem of circulant matrices, is applicable for stationary geostatistical models when the grid discretization, in each spatial direction, is uniform in the volume of interest. We also used a structural approach to jointly invert cross-hole electrical resistance and ground-penetrating radar traveltime data in three dimensions. The two models are coupled by assuming, at all points, that the cross product of the gradients of the two models is zero. No petrophysical relationship between electrical conductivity and relative permittivity is assumed but is instead obtained as a by-product of the inversion. The approach has been applied to data collected in a U.K. sandstone aquifer in order to improve characterization of the vadose zone hydrostratigraphy. By analyzing scatterplots of electrical conductivity versus relative permittivity together with petrophysical models a zonation could be obtained with corresponding estimates of the electrical formation factor, the water content, and the effective grain radius of the sediments. The approach provides greater insight into the hydrogeological characteristics of the subsurface than by using conventional geophysical inversion methods.

Citation: Linde, N., A. Binley, A. Tryggvason, L. B. Pedersen, and A. Revil (2006), Improved hydrogeophysical characterization using joint inversion of cross-hole electrical resistance and ground-penetrating radar traveltime data, Water Resour. Res., 42, W12404, doi:10.1029/2006WR005131.

\section{Introduction}

[2] Combinations of cross-hole tomographic techniques (e.g., ground-penetrating radar (GPR), electrical resistance tomography (ERT), and seismics) are increasingly used in hydrogeological site characterization [e.g., Hubbard et al., 2001; Binley et al., 2002a, 2002b; Tronicke et al., 2004]. In order to produce a hydrogeological model, different geophysical data types are typically inverted individually and, at a later stage, combined with other geophysical models and available hydrogeological data [e.g., Hubbard et al., 2001].

\footnotetext{
${ }^{1}$ Department of Earth Sciences/Geophysics, Uppsala University, Uppsala, Sweden.

${ }^{2}$ Department of Hydrogeophysics and Porous Media, Centre Européen de Recherche et d'Enseignement des Géosciences de l'Environnement, Centre National de la Recherche Scientifique, Université Paul Cézanne, Aix-en-Provence, France.

${ }^{3}$ Now at Institute of Geophysics, Swiss Federal Institute of Technology Hönggerberg, Zurich, Switzerland.

${ }^{4}$ Department of Environmental Science, Lancaster University, Lancaster, UK.
}

Copyright 2006 by the American Geophysical Union. 0043-1397/06/2006WR005131\$09.00
[3] Geophysical inverse problems are often regularized because of noise in the data and the mixed determined character of most inverse problems [e.g., Menke, 1984]. In this paper, we define regularization operators that are consistent with available borehole data and geological understanding; thereby, constructing models of physical properties that are more closely related to the underlying geology compared with models obtained from inversion schemes that use traditional regularization operators, such as damping [Marquardt, 1970] or Tikhonov regularization [Tikhonov and Arsenin, 1977]. We show that when the grid discretization is uniform in each spatial direction, stochastic regularization operators can be calculated efficiently for a given stationary geostatistical model by combining circulant embedding of the model covariance matrix [e.g., Dietrich and Newsam, 1997] and the diagonalization theorem of circulant matrices [e.g., Golub and Van Loan, 1996].

[4] Another feature of geophysical inverse problems is that the resolution of the resulting models varies throughout the model and that the patterns of resolution variations are different for different geophysical techniques [e.g., Day-Lewis et al., 2005]. For example, models based on cross-hole ERT where the electrical resistance is measured by a transmission of currents implanted at the ground surface or in boreholes [e.g., LaBrecque et al., 1996] have the highest resolution close to the boreholes. On the other 
hand, models that are based on inversion of multioffset gather (MOG) GPR traveltimes that represent the times it take for the first arrivals of high-frequency electromagnetic pulses transmitted from different depths in one borehole to travel to different depths in other boreholes [e.g., Peterson, 2001] have the highest resolution in the central part of the tomogram [e.g., Day-Lewis et al., 2005]. It should therefore be possible to improve the resulting models by jointly inverting cross-hole ERT and GPR data.

[5] Joint inversion of geophysical data has received considerable attention within the geophysical community. Geophysical data that are sensitive to the same physical quantity, for example, magnetotelluric and DC resistivity data [Vozoff and Jupp, 1975], can be simultaneously inverted by minimizing an objective function that includes the data misfit of the different data types and the most difficult task is how to determine the relative weighting of the different data types [Lines et al., 1988]. When collecting data that are sensitive to different physical quantities, it might be possible to perform joint inversion by mildly constraining the solutions around an assumed petrophysical relationship, for example, a ratio of $P$ and $S$ wave traveltimes [e.g., Tryggvason et al., 2002]. However, petrophysical links between geophysical properties, at a specific site, are in many cases unknown as they are affected by a multitude of rock properties and associated state variables [e.g., Mavko et al., 1998; Schön, 1996]. The problem is further complicated by measurement errors and the resolution loss that is inherent when solving inverse problems [e.g., Day-Lewis and Lane, 2004; Day-Lewis et al., 2005; Linde et al., 2006].

[6] One approach to jointly invert geophysical data when the site-specific petrophysical relationship is unknown, such as for gravity and seismic data, is to assume interfaces (i.e., geological boundaries) at the same locations, but different and unlinked model parameterizations within each model block [Lines et al., 1988]. Such an approach is appropriate when inverting for layered models. However, most inversion algorithms use a fine model discretization where unique solutions are found by imposing smoothness constraints [e.g., Constable et al., 1987], damping constraints [e.g., Marquardt, 1970], or a priori information [e.g., Musil et al., 2003]; therefore the method proposed by Lines et al. [1988] is often not applicable.

[7] An approach that is better suited to overparameterized problems is the structural approach [Haber and Oldenburg, 1997; Gallardo and Meju, 2003, 2004; Tryggvason and Linde, 2006]. The idea behind the structural approach is that geophysical properties in the near surface are dependent on the same underlying geology and pore water. It is therefore reasonable to assume that changes in different physical properties, at a given position, occur in the same direction. Gallardo and Mејu [2003] inverted surface based DC resistivity and refraction seismic data in two dimensions under the constraint that the cross products of the gradients of the model vectors, termed the cross-gradients function by Gallardo and Мeju [2003], are zero. We adopt a similar approach to simultaneously invert cross-hole ERT and MOG GPR traveltime data in three dimensions. The principle differences between our inversion method and the one presented by Gallardo and Мејu [2003, 2004] are the different geophysical methods used, that we perform three-dimensional inversion instead of two-dimensional inversion, that the cross-gradients function provides soft constraints and not hard constraints, that our regularization operators are based on geostatistical models and that the weight given to the regularization term vary during the inversion process, and that we use an iterative equation solver compared with a direct solver that makes it possible to solve realistic three-dimensional problems.

[8] The stochastic regularization operators and our implementation of the structural approach to joint inversion were tested with data collected in unsaturated Sherwood Sandstone collected close to Eggborough, North Yorkshire, UK [e.g., Binley et al., 2002a; West et al., 2003; Cassiani and Binley, 2005; Binley et al., 2005].

[9] This paper has the following goals: (1) to compare results from individual inversions based on stochastic regularization with models obtained from inversion with regularization based on traditional smoothness constraints; (2) to demonstrate that joint inversion of cross-hole ERT and MOG GPR traveltime data improves the final tomograms, and hence aquifer zonation, compared with tomograms obtained from individual inversions [e.g., Tronicke et al., 2004]; and (3) finally to use petrophysical models to estimate possible values of the electrical formation factor, water content, and effective grain radius within each zone.

\section{Inversion Method}

\subsection{Formulation of the Inverse Problem}

[10] Geophysical inverse problems are often nonlinear and significantly more model parameters are typically used than the number of model parameters that can be constrained by the geophysical data only. Nonlinear inverse problems are commonly solved iteratively where the inverse problem is linearized around the model of the previous iteration and where the sensitivities of the model parameters with respect to the data (i.e., the Jacobian or sensitivity matrix) are used to find an updated model with improved data fit. Unique solutions are found by regularizing the inverse problem, which is typically achieved by assuming that model parameters are strongly correlated in space or that the final model is close to an a priori model, which is the approach chosen in this work. General literature on classical discrete geophysical inverse theory includes the work by Menke [1984] and Parker [1994]. The inversion method presented in this paper is developed in this tradition.

[11] A different approach to the geophysical inverse problem is to search for all possible models that honor the data and available a priori information. These problems are typically solved with Monte Carlo methods [e.g., Mosegaard and Tarantola, 1995], but they are still computationally infeasible for the application considered here and many other applications of interest.

[12] Cokriging has been an influential method to solve hydrological inverse problems where the relationship between the model parameters and the data (i.e., the forward problem) is linearized [Kitanidis and Vomvoris, 1983]. Weakly nonlinear problems can be solved with an iterative procedure that solves the cokriging equations at each iteration [Yeh et al., 1996] and applications to geophysics have been reported [Yeh et al., 2002]. 
[13] Our inversion method is a regularized least squares algorithm that is closely related to Occam's inversion [Constable et al., 1987]. In contrast to Occam's inversion, we define the regularization operator based on a stochastic model [Maurer et al., 1998]. We estimate the stochastic models by using EM conductivity logs collected in the boreholes for the ERT inversion and zero-offset profiles (ZOP) of GPR traveltimes where the transmitting and receiving antennas are located at the same depth for the GPR inversion. In addition, we look for geophysical models that are structurally similar by penalizing deviations from structural similarity, which we quantify with the crossgradients function [Gallardo and Meju, 2003]. At each iteration, we solve the linearized inverse problem with the iterative conjugate gradient algorithm LSQR [Paige and Saunders, 1982].

\subsection{Inversion of ERT Data}

[14] The following system of equations describing the ERT inversion is to be satisfied in a least squares sense, where the upper terms in the concatenated system of equations correspond to the expected data fit for the updated model obtained by linearization around the previous model and the lower terms correspond to regularization that avoids excessive and uncorrelated variations between neighboring model parameters [e.g., Paige and Saunders, 1982; Menke, 1984; Constable et al., 1987; LaBrecque et al., 1996; Siripunvaraporn and Egbert, 2000]:

$$
\begin{aligned}
& {\left[\begin{array}{c}
\varepsilon_{p}^{\sigma}\left(\mathbf{C}_{\mathrm{d}}^{\sigma}\right)^{-0.5} \mathbf{J}_{p}^{\sigma} \\
\left.\left(\mathbf{C}_{\mathrm{m}}^{\sigma}\right)^{-0.5}\right]
\end{array}\right]\left[\mathbf{m}_{p+1}^{\sigma}\right]} \\
& =\left[\begin{array}{cc}
\varepsilon_{p}^{\sigma}\left(\mathbf{C}_{\mathrm{d}}^{\sigma}\right)^{-0.5}\left(\mathbf{d}^{\sigma}-\mathbf{F}^{\sigma}\left(\mathbf{m}_{p}^{\sigma}\right)+\mathbf{J}_{p}^{\sigma} \mathbf{m}_{p}^{\sigma}\right) \\
\left(\mathbf{C}_{\mathbf{m}}^{\sigma}\right)^{-0.5} \mathbf{m}_{\text {apriori }}^{\sigma}
\end{array}\right],
\end{aligned}
$$

where $\mathbf{m}_{p+1}^{\sigma}, \mathbf{m}_{p}^{\sigma}$, and $\mathbf{m}_{\text {apriori }}^{\sigma}$ are the model vectors at the present iteration (i.e., iteration $p+1$ ), the previous iteration (i.e., iteration $p$ ), and the a priori model, respectively. Each model vector consists of $M^{\sigma}$ elements of the natural logarithm of electrical conductivity $(\mathrm{S} / \mathrm{m}) ; \mathbf{d}^{\sigma}$ is the data vector of $N^{\sigma}$ observed resistances $(\mathrm{Ohm}) ; \mathbf{J}_{p}^{\sigma}$ is the Jacobian matrix with elements $J_{p}^{\sigma}(i, j)=\frac{\partial d^{\sigma}(i)}{\partial m^{\sigma}(j)} ; \mathbf{C}_{d}^{\sigma}$ is the corresponding data covariance matrix, which is diagonal under the assumption that data errors are uncorrelated, and may be estimated by performing reciprocal measurements [e.g., LaBrecque et al., 1996]; $\mathbf{F}^{\sigma}\left(\mathbf{m}_{p}^{\sigma}\right)$ generates a vector of the computed resistances (i.e., the forward response); $\varepsilon_{p}^{\sigma}$ is a trade-off parameter between data fit and model structure; and $\mathbf{C}_{m}^{\sigma}$ is the model covariance matrix. For each iteration, a line search [e.g., Siripunvaraporn and Egbert, 2000] is performed to find the value of $\varepsilon_{p}^{\sigma}$ that minimizes the data misfit. The inversion process terminates when the target data misfit is reached or when no additional improvement in data misfit is obtained from one iteration to the next.

[15] Our numerical solution of the forward problem, $\mathbf{F}^{\sigma}\left(\mathbf{m}_{p}^{\sigma}\right)$, uses the Lancaster University 3D finite element code R3 [Binley, 2005]. Uniform resistivity is assigned within each linear brick type element in the finite element mesh. The model computes the voltage field resulting from point source current injection at each current electrode location. Computation of the voltage measurement for each four electrode configuration is then achieved using the principle of superposition. The algorithm utilizes a diagonally scaled conjugate gradient linear equation solver to minimize computer storage requirement. Calculation of the Jacobian, $\mathbf{J}_{p}^{\sigma}$, is computed using the principle of reciprocity [Geselowitz, 1971].

\subsection{Inversion of MOG GPR Traveltime Data}

[16] A corresponding system of equations describing the traveltime inversion is to be satisfied in a least squares sense:

$$
\begin{aligned}
& {\left[\begin{array}{c}
\varepsilon_{\mathrm{p}}^{\mathrm{s}}\left(\mathbf{C}_{\mathrm{d}}^{\mathrm{s}}\right)^{-0.5} \mathbf{J}_{\mathrm{p}}^{\mathrm{s}} \\
\left(\mathbf{C}_{\mathbf{m}}^{\mathrm{s}}\right)^{-0.5}
\end{array}\right]\left[\mathbf{m}_{\mathrm{p}+1}^{\mathrm{s}}\right]} \\
& =\left[\begin{array}{c}
\varepsilon_{\mathrm{p}}^{\mathrm{s}}\left(\mathbf{C}_{\mathrm{d}}^{\mathrm{s}}\right)^{-0.5}\left(\mathbf{d}^{\mathrm{s}}-\mathbf{F}_{p}^{\mathrm{s}}\left(\mathbf{m}_{p}^{\mathrm{s}}\right)+\mathbf{J}_{p}^{\mathrm{s}} \mathbf{m}_{p}^{\mathrm{s}}\right) \\
\left(\mathbf{C}_{\mathbf{m}}^{\mathrm{s}}\right)^{-0.5} \mathbf{m}_{\text {apriori }}^{\mathrm{s}}
\end{array}\right]
\end{aligned}
$$

where $\mathbf{m}_{p+1}^{\mathrm{s}}, \mathbf{m}_{p}^{\mathrm{s}}$, and $\mathbf{m}_{\text {apriori }}^{\mathrm{s}}$ are the model vectors of the present iteration, the previous iteration, and the a priori model, respectively. Each model vector consists of $M^{\mathrm{s}}$ elements of the slowness $s(\mathrm{~s} / \mathrm{m}) ; \mathbf{d}^{\mathrm{s}}$ is the data vector of $N^{s}$ observed traveltimes (s); $\mathbf{J}_{p}^{\mathrm{s}}$ is the Jacobian matrix with elements $J_{p}^{\mathrm{s}}(i, j)=\frac{\partial d^{s}(i)}{\partial m^{s}(j)} ; \mathbf{C}_{\mathrm{d}}^{\mathrm{s}}$ is the corresponding data covariance matrix and its accurate estimation is an open question, but its entries should be chosen larger than the errors associated with the picking of the first arrivals to incorporate errors associated with mislocations of boreholes and simplified forward models; $\mathbf{F}_{p}^{\mathrm{s}}\left(\mathbf{m}_{p}^{\mathrm{s}}\right)$ are the computed traveltimes (i.e., the forward response); $\varepsilon_{p}^{s}$ is a trade-off parameter between data fit and model structure that is found in the same way as described for the ERT inversion (see section 2.2); and $\mathbf{C}_{\mathrm{m}}^{\mathrm{s}}$ is the model covariance matrix.

[17] The forward modeling, $\mathbf{F}_{p}^{\mathrm{s}}\left(\mathbf{m}_{p}^{\mathrm{s}}\right)$, and computation of the Jacobian, $\mathbf{J}_{p}^{\mathrm{s}}$, are based on the nonlinear traveltime tomography algorithm PStomo_eq [Tryggvason et al., 2002]. The first arrival traveltimes are computed using the finite difference (FD) algorithm time3d of Podvin and Lecomte [1991]. This algorithm is based on the first-order approximation to the Eikonal equation. Podvin and Lecomte [1991] claimed that time3d could handle velocity contrasts as high as 1:10, which is sufficient for all MOG GPR applications. Ray tracing is performed by a posteriori back propagation perpendicular to the wavefronts from the receivers to the transmitters [Hole, 1992]. The forward response represents the first arrivals of a given model regardless of if the first arrivals correspond to a direct, refracted, or reflected wave. The inverse problem is nonlinear because the raypaths depend on the slowness structure. Thus, if the slowness structure is changed, the raypaths will change as well. Therefore new raypaths must be computed after each iteration. Assuming the slowness structure can be represented by cells of constant slowness, the elements in the Jacobian, $\mathbf{J}_{p}^{\mathrm{s}}$, are simply the ray length in each cell.

\subsection{Joint Inversion of ERT and MOG GPR Traveltimes}

[18] We jointly invert the ERT and GPR traveltime data by enforcing structural similarity quantified by the cross- 
gradients function. The cross-gradients function at the previous iteration is defined as [Gallardo and Meju, 2003]:

$$
\mathbf{t}_{p}(i, j, k)=\nabla \mathbf{m}_{p}^{\sigma}(i, j, k) \times \nabla \mathbf{m}_{p}^{\mathrm{s}}(i, j, k)
$$

where the indices $i, j$, and $k$ define the model parameters (i.e., the logarithm of electrical conductivity and radar slowness, respectively) in the $x, y$, and $z$ directions, respectively. Both $\mathbf{m}_{p}^{\sigma}$ and $\mathbf{m}_{p}^{\mathrm{s}}$ are stored as vectors, and the indices in the parentheses indicate the elements to operate upon, whereas the actual location in the model vectors are dependent on the scheme used to store the mesh. If all three components of the cross-gradients function is zero for a given element, it implies that the gradients of the two models point in the same or opposite direction, or that the gradients of one of the models are zero. The magnitude of the cross-gradients function is unchanged if calculated for reciprocal model properties (e.g., electrical conductivity or electrical resistivity, slowness or velocity). It is recommended to invert for the logarithm of the geophysical properties that vary widely in space (such as electrical conductivity) to ensure that changes in regions of high and low values of the geophysical property of interest are given equal importance. The discretized formulation of the crossgradients function using a forward difference scheme is

$$
\begin{aligned}
t_{p}^{x}(i, j, k)= & \frac{1}{\Delta y \Delta z}\left(m^{\sigma}(i, j+1, k)-m^{\sigma}(i, j, k)\right)\left(m^{s}(i, j, k+1)\right. \\
& \left.-m^{s}(i, j, k)\right)-\frac{1}{\Delta y \Delta z}\left(m^{\sigma}(i, j, k+1)\right. \\
& \left.-m^{\sigma}(i, j, k)\right)\left(m^{s}(i, j+1, k)-m^{s}(i, j, k)\right)
\end{aligned}
$$

$$
\begin{aligned}
t_{p}^{y}(i, j, k)= & \frac{1}{\Delta x \Delta z}\left(m^{\sigma}(i, j, k+1)-m^{\sigma}(i, j, k)\right)\left(m^{s}(i+1, j, k)\right. \\
& \left.-m^{s}(i, j, k)\right)-\frac{1}{\Delta x \Delta z}\left(m^{\sigma}(i+1, j, k)\right. \\
& \left.-m^{\sigma}(i, j, k)\right)\left(m^{s}(i, j, k+1)-m^{s}(i, j, k)\right),
\end{aligned}
$$

$$
\begin{aligned}
t_{p}^{z}(i, j, k)= & \frac{1}{\Delta x \Delta y}\left(m^{\sigma}(i+1, j, k)-m^{\sigma}(i, j, k)\right)\left(m^{s}(i, j+1, k)\right. \\
& \left.-m^{s}(i, j, k)\right)-\frac{1}{\Delta x \Delta y}\left(m^{\sigma}(i, j+1, k)\right. \\
& \left.-m^{\sigma}(i, j, k)\right)\left(m^{s}(i+1, j, k)-m^{s}(i, j, k)\right),
\end{aligned}
$$

where the superscripts indicate the different components of the cross-gradients function and $\Delta x, \Delta y$ and $\Delta z$ are the model discretization distances. The elements of the cross-gradients function (see equations (4)-(6)) are stored into the three vectors $\mathbf{t}_{p}^{x}, \mathbf{t}_{p}^{y}$, and $\mathbf{t}_{p}^{z}$. The cross-gradients function for the present iteration is estimated by a firstorder Taylor expansion around the cross-gradients function at the previous iteration (note that Gallardo and Мејu [2004] linearize around their a priori models), shown here for

$$
\mathbf{t}_{p+1}^{\mathrm{x}} \cong \mathbf{t}_{p}^{x}+\mathbf{B}_{p}^{x}\left(\begin{array}{c}
\mathbf{m}_{p+1}^{\sigma}-\mathbf{m}_{p}^{\sigma} \\
\mathbf{m}_{p+1}^{\mathrm{s}}-\mathbf{m}_{p}^{\mathrm{s}}
\end{array}\right)
$$

where $\mathbf{B}_{p}^{x}$ is the Jacobian of the cross-gradients function in the $x$ direction with regards to the model parameters. Every row of $\mathbf{B}_{p}^{x}$ has six nonzero elements that can easily be derived from equation (4) (see also equation (9) of Gallardo and Meju [2004]).

[19] In order to enforce structural similarity between $\mathbf{m}_{p+1}^{\sigma}$ and $\mathbf{m}_{p+1}^{\mathrm{s}}$, equations (1)-(7) are combined to a system of equations to minimize in a least squares sense similarly to equations (1) and (2):

$$
\left[\begin{array}{c}
\left(\mathbf{C}_{\mathrm{d}}^{\epsilon}\right)^{-0.5} \mathbf{J}_{p} \\
\mathbf{C}_{\mathbf{m}}^{-0.5} \\
\lambda \mathbf{B}_{p}^{x} \\
\lambda \mathbf{B}_{p}^{y} \\
\lambda \mathbf{B}_{p}^{z}
\end{array}\right]\left[\mathbf{m}_{p+1}\right]=\left[\begin{array}{c}
\left(\mathbf{C}_{d}^{\epsilon}\right)^{-0.5}\left(\mathbf{d}-\mathbf{F}_{p}\left(\mathbf{m}_{p}\right)+\mathbf{J}_{p} \mathbf{m}_{p}\right) \\
\mathbf{C}_{\mathbf{m}}^{-0.5} \mathbf{m}_{\text {apriori }} \\
\lambda\left(\mathbf{B}_{p}^{x} \mathbf{m}_{p}-\mathbf{t}_{p}^{x}\right) \\
\lambda\left(\mathbf{B}_{p}^{y} \mathbf{m}_{p}-\mathbf{t}_{p}^{y}\right) \\
\lambda\left(\mathbf{B}_{p}^{z} \mathbf{m}_{p}-\mathbf{t}_{p}^{z}\right)
\end{array}\right]
$$

where

$$
\begin{aligned}
& \mathbf{m}_{p+1}=\left[\begin{array}{l}
\mathbf{m}_{p+1}^{\sigma} \\
\mathbf{m}_{p+1}^{s}
\end{array}\right], \mathbf{m}_{p}=\left[\begin{array}{l}
\mathbf{m}_{p}^{\sigma} \\
\mathbf{m}_{p}^{s}
\end{array}\right], \mathbf{m}_{\text {apriori }}=\left[\begin{array}{l}
\mathbf{m}_{\text {apriori }}^{\sigma} \\
\mathbf{m}_{\text {apriori }}^{s}
\end{array}\right], \\
& \mathbf{d}=\left[\begin{array}{c}
\mathbf{d}^{\sigma} \\
\mathbf{d}^{s}
\end{array}\right], \mathbf{F}_{p}\left(\mathbf{m}_{p}\right)=\left[\begin{array}{c}
\mathbf{F}^{\sigma}\left(\mathbf{m}_{p}^{\sigma}\right) \\
\mathbf{F}_{p}^{s}\left(\mathbf{m}_{p}^{s}\right)
\end{array}\right] \\
& \left(\mathbf{C}_{d}^{\varepsilon}\right)^{-0.5}=\left[\begin{array}{cc}
\varepsilon_{p}^{\sigma}\left(\mathbf{C}_{d}^{\sigma}\right)^{-0.5} & 0 \\
0 & \varepsilon_{p}^{s}\left(\mathbf{C}_{d}^{s}\right)^{-0.5}
\end{array}\right], \\
& \mathbf{C}_{\mathrm{m}}^{-0.5}=\left[\begin{array}{cc}
\left(\mathbf{C}_{\mathrm{m}}^{\sigma}\right)^{-0.5} & 0 \\
0 & \left(\mathbf{C}_{\mathrm{m}}^{s}\right)^{-0.5}
\end{array}\right],
\end{aligned}
$$

and $\lambda$ is a large and constant weighting parameter that ensures that the cross-gradients function of the resulting models is close to zero. The upper terms in equation (8) represent the data fit for both the ERT and GPR data, the second term represents the regularization terms for the two data types, and the last three terms represent the crossgradients constraints in the $x, y$, and $z$ spatial directions, respectively. Note that it would be possible to incorporate known linear correlations between different geophysical model parameters by adding nonzero values in the offdiagonal blocks of $\mathbf{C}_{\mathrm{m}}^{-0.5}$. Equation (8) (as well as equations (1) and (2) for the individual inversions) is solved with the LSQR algorithm [Paige and Saunders, 1982]. LSQR is a conjugate gradient method where the normal equations are not formed, thereby, preserving the condition number of equation (8). A preconditioner is applied that ensures that the norm of each column of the matrix on the left-hand side of equation (8) is unity, which avoids unnecessary ill conditioning [Paige and Saunders, 1982]. An iterative solution of equation (8) is suitable because we solve three-dimensional problems where the dimension of equation (8) is large and computer storage becomes a limiting factor. The sparseness of equation (8) is utilized by using sparse solvers.

[20] In a first stage, we perform separate inversions of the ERT and the GPR traveltime data (see sections 2.2 and 2.3) to determine standard deviations of the data for which 
solutions with a weighted RMS of 1 can be found. This procedure ensures an even weighting of the two data types. The individual inversions also serve evaluation purposes. If no jointly inverted models (see equation (8)) with weighted RMS values of 1 or lower can be obtained, it suggests that the constraints based on the cross-gradients function are physically invalid for that application.

\subsection{Petrophysical Basis for the Joint Inversion Method}

[21] It is possible to perform joint inversion with crossgradients constraints by assuming that the geometry and properties of lithological units control the model parameters [e.g., Gallardo and Meju, 2004]. However, this assumption is not valid in many applications and we argue that it is necessary to use petrophysical models to identify the controlling rock properties and state variables influencing the model parameters and, at a later stage, define under what conditions the joint inversion scheme is likely to be valid.

[22] We consider a partially saturated sand/siltstone and the expected relationship between relative permittivity and electrical conductivity in such a system. This was done in order to understand under what conditions joint inversion of cross-hole ERT and GPR traveltimes with cross-gradients constraints are valid when applied to unsaturated sandstone and to provide a petrophysical basis to evaluate the resulting tomograms. The radar slowness, $s$, and the effective relative permittivity, $\kappa_{\text {eff }}$, are for low-loss materials related as $\kappa_{\text {eff }}=s^{2} \mathrm{c}^{2}$, where $\mathrm{c}$ is $3 \times 10^{8} \mathrm{~m} / \mathrm{s}$. We used the inverse of the electrical formation factor instead of porosity when deriving the volume averaging equations (see Appendix A). The inverse of the electrical formation factor is a good estimate of the effective porosity [e.g., Revil and Cathles, 1999], i.e., it is a relevant parameter to model transport properties through the connected pore volume. The effective relative permittivity, $\kappa_{\text {eff }}$, is given by

$$
\kappa_{\text {eff }}=\frac{1}{F}\left[S_{w}^{n} \kappa_{w}+\left(1-S_{w}^{n}\right) \kappa_{a}+(F-1) \kappa_{s}\right]
$$

where $\kappa_{w}, \kappa_{a}$, and $\kappa_{s}$ are the relative permittivities of water, air, and the minerals forming the rock matrix, respectively; $F$ is the electrical formation factor, $S_{w}$ is the water saturation, and $n$ is Archie's second exponent [e.g., Waxman and Smits, 1968]. In the following, we adopt the common practice of assuming that $\kappa_{w}, \kappa_{a}$, and $\kappa_{s}$ are constant within the volume of interest.

[23] The electrical conductivity of the air and the mineral grains can be safely neglected, but surface conduction in the electrical double layer coating the grains can be significant [e.g., Revil and Glover, 1998]. The resulting equation for the effective electrical conductivity, $\sigma_{\text {eff }}(\mathrm{S} / \mathrm{m})$ is

$$
\sigma_{\text {eff }}=\frac{1}{F}\left[S_{w}^{n} \sigma_{w}+(F-1) \sigma_{s}\right]
$$

where $\sigma_{w}(\mathrm{~S} / \mathrm{m})$ is the electrical conductivity of the pore water and $\sigma_{s}(\mathrm{~S} / \mathrm{m})$ is the surface conduction. For a granular material with uniform grain radius, $R(\mathrm{~m})$, the surface conductivity entering equation (11) is

$$
\sigma_{s}=\frac{3}{R} \sum_{s}
$$

where $\Sigma_{s}(\mathrm{~S})$ is called the specific surface conductivity and it is fairly constant in relation to $R$ [e.g., Leroy and Revil, 2004]. For nonuniform grain distributions it is necessary to replace $R$ with an effective grain radius, $R_{\text {eff }}(\mathrm{m})$, e.g., by taking the harmonic mean of the grain size distribution weighted by their relative fractions.

[24] The petrophysical models given in equations (10)(12) are simplified models that include the dominant factors that are likely to control the electrical and dielectric properties of unsaturated sandstone. Alternative models are present in the literature and more elaborate models may have a higher predictive value but are likely to include parameters that are poorly known in field applications.

\subsubsection{Saturated Media}

[25] In saturated media, equations (10) and (11) simplify to

$$
\begin{aligned}
& \kappa_{\text {eff }}=\frac{1}{F}\left[\kappa_{w}+(F-1) \kappa_{s}\right], \\
& \sigma_{\text {eff }}=\frac{1}{F}\left[\sigma_{w}+(F-1) \sigma_{s}\right] .
\end{aligned}
$$

[26] Variations in $\kappa_{\text {eff }}$ are only affected by variations in $F$, whereas variations in $\sigma_{\text {eff }}$ can be attributed to variations in either $F, \sigma_{w}$, or $\sigma_{s}$. Minimization of the cross-gradients function is for a random media (e.g., described by exponential space random functions) valid only if $\sigma_{s}$ and $\sigma_{w}$ are strongly correlated with $F$ or if $\sigma_{s}$ can be neglected or assumed to be constant and $\sigma_{w}$ is constant. If these conditions are not fulfilled, we have to assume that the Earth structure is composed of fairly homogeneous zones, where any variations in rock properties from one zone to another occurs at the same location, which is the justification for the method presented by Gallardo and Meju [2003]. This is a reasonable assumption when the subsurface consists of fairly distinct units, but is not valid for materials that are better described as continuous random fields. In this case, imposing a near-zero cross-gradients function will remove small-scale features. Because of resolution limitations of geophysical methods, this is not necessarily a severe limitation, because we cannot expect to resolve small-scale variability anyway. However, minimization of the crossgradients function is clearly inappropriate in applications where variations of $\sigma_{w}$ are varying gradually in a different direction than the normal to geological boundaries. This would be expected for many applications at contaminated sites, for instance, mapping of saline contaminant plumes.

\subsubsection{Unsaturated Media}

[27] In unsaturated media, the problem is further complicated by the influence of the water saturation, $S_{w}$, and minimizing the cross-gradients function is meaningful only if changes in $S_{w}^{n}$ is confined within fairly homogeneous geological units or if the gradients of $S_{w}^{n}$ are normal to geological boundaries (e.g., clay formations have a higher water saturation than sand formations). This is often expected because the capillary pressure is sensitive to grain size. The same reasoning applies both to $\sigma_{w}$ or $\sigma_{s}$. However, $F$ can vary freely.

\subsection{Calculation of Stochastic Regularization Operators}

[28] Regularization through smoothness operators [e.g., Constable et al., 1987] give models with strong spatial 
correlations and regularization through damping operators [Marquardt, 1970] give models that are close to an initial model, but with no spatial correlation. Maurer et al. [1998] showed that regularization of inverse problems based on a stochastic regularization operator, $\mathbf{C}_{\mathrm{m}}^{-0.5}$ (e.g., equation (1)), can be expressed as a combination of generalized smoothness and damping regularization operators, resulting in geologically more reasonable models that are consistent with geostatistical descriptions of geological media. Maurer et al. [1998] also proposed a method to generate stochastic regularization operators based on the von Kármán autocovariance function and determined $\mathbf{C}_{\mathrm{m}}^{-0.5}$ by a grid search method. Maurer et al. [1998] applied their method to a synthetic two-dimensional cross-hole tomographic seismic experiment.

[29] In most applications, only smoothness or damping constraints are used because the calculation of $\mathbf{C}_{\mathrm{m}}^{-0.5}$ under general conditions is for many three-dimensional applications prohibitively computationally expensive. Furthermore, the model covariance matrix, $\mathbf{C}_{\mathrm{m}}$, is poorly known in most geophysical applications. However, good approximations of $\mathbf{C}_{\mathrm{m}}$ can sometimes be obtained in nearsurface applications, where borehole geophysical logs can be used to infer the spatial correlation structure of physical properties. However, the computational problems of computing $\mathbf{C}_{\mathrm{m}}^{-0.5}$ remain.

[30] There are conditions under which $\mathbf{C}_{\mathrm{m}}^{-0.5}$ can be calculated efficiently and with small computing storage requirements. The model covariance matrix $\mathbf{C}_{\mathrm{m}}$ is a symmetrical Toeplitz matrix if the correlation function is stationary and the grid discretization is uniform in each direction [e.g., Dietrich and Newsam, 1997]. A Toeplitz matrix has constant values along diagonals in each block and a symmetrical Toeplitz matrix is therefore defined completely by its first column. Dietrich and Newsam [1997] used circulant embedding of Toeplitz model covariance matrices into nonnegative circulant matrices when they developed a computationally efficient method to generate random Gaussian fields with a given correlation function. Nowak et al. [2003] used circulant embedding of model covariance matrices to efficiently perform matrix-matrix computations arising in geostatistics (e.g., in cokriging). Here, we use circulant embedding and the diagonalization theorem for circulant matrices to compute the stochastic regularization operator, $\mathbf{C}_{\mathrm{m}}^{-0.5}$, in a computationally efficient way.

[31] Following Dietrich and Newsam [1997], $\mathbf{C}_{\mathrm{m}}$ of a stationary process $Y(x)$ with correlation function $r(x)$ sampled on the uniform 1D mesh $\Omega=\left\{x_{0}, \ldots, x_{m}\right\}$ has entries $\mathbf{C}_{m}(p, q)=r\left(\left|x_{p}-x_{q}\right|\right)$. A model covariance matrix $\mathbf{C}_{\mathrm{m}}$ of size $m \times m$ can be circulantly embedded into a symmetric circulant matrix $\mathbf{S}$ of size $2 M \times 2 M$ by assigning the following entries to the first column $\mathbf{s}$ of $\mathbf{S}$

$$
\begin{array}{ll}
s_{k}=r_{k}, & k=0, \ldots, m, \\
s_{2 M-k}=r_{k}, & k=1, \ldots, m-1,
\end{array}
$$

where if $M>m$ the entries $s_{m+1}, \ldots, s_{2 M-m}$ are arbitrary or conveniently chosen. The next column of $\mathbf{S}$ can be obtained by shifting the first column circularly, i.e., such that the last element becomes first and all other elements are shifted forward by one, and so on. Being circulant, S, can be decomposed by using the diagonalization theorem of circulant matrices

$$
\mathbf{S}=\frac{1}{2 M} \mathbf{F} \Lambda \mathbf{F}^{H},
$$

where $\mathbf{F}$ is the standard fast Fourier transform (FFT) matrix of size $2 M$ with entries $F_{p q}=\exp (2 \pi i q r / 2 M), \mathbf{F}^{\mathrm{H}}$ is the conjugate transpose of $\mathbf{F}$, and $\boldsymbol{\Lambda}$ is a diagonal matrix whose diagonal entries form the vector $\tilde{\mathbf{s}}=$ Fs [e.g., Golub and Van Loan, 1996]. The matrix $\mathbf{S}$ is nonnegative definite if all entries of $\tilde{\mathbf{s}}$ are nonnegative. These results have been extended to two and three dimensions; see Ranguelova [2002] for details.

[32] The matrix $\mathbf{S}^{-1 / 2}$ is for the one-dimensional case also circulant and its first column can be obtained as $\mathbf{F}^{\mathrm{H}} \tilde{\mathbf{s}}^{-1 / 2}$ and the entries corresponding to the first column of $\mathbf{C}_{\mathrm{m}}^{-0.5}$ can be retrieved from entries 1 to $m$ (see equation (15)); all other columns of $\mathbf{C}_{\mathrm{m}}^{-0.5}$ can be calculated by shifting the first column circularly. In order to decrease memory requirements, we only store elements of $\mathbf{C}_{\mathrm{m}}^{-0.5}$ that are larger than $1 \%$ of the maximum value of $\mathbf{C}_{\mathrm{m}}^{-0.5}$. In three dimensions, the only difference is that we need to express $\mathbf{s}$ and $\tilde{\mathbf{s}}$ as three-dimensional arrays and apply three-dimensional FFT. Our method to calculate $\mathbf{C}_{\mathrm{m}}^{-0.5}$ is computationally efficient because we perform operations on a vector $\mathbf{s}$ instead of the model covariance matrix $\mathbf{C}_{\mathrm{m}}$.

[33] Dietrich and Newsam [1997] proved the existence of nonnegative definite matrices $\mathbf{S}$ for different types of embedded covariance matrices and dimensions; they also provided numerical results concerning the necessary size of the matrix $\mathbf{S}$ in relation to the integral scales of the underlying covariance model for one- and two dimensional domains. In order to ensure that $\mathbf{S}$ is nonnegative definite in three dimensions when using an exponential correlation function, we have found that it is necessary to choose $M$ to be at least seven integral scales in each direction and choosing $s_{m+1}, \ldots, S_{2 M-m}$ to be the corresponding values of $r(l)$. The exponential correlation function is for a stationary three-dimensional domain defined as

$$
r(l)=c e^{-l}
$$

where $c$ is the variance, e is the natural logarithm, and $l$ is defined as

$$
l=\sqrt{\left(\frac{h_{x}}{I_{x}}\right)^{2}+\left(\frac{h_{y}}{I_{y}}\right)^{2}+\left(\frac{h_{z}}{I_{z}}\right)^{2}}
$$

where $h_{x}(\mathrm{~m}), h_{y}(\mathrm{~m})$, and $h_{z}(\mathrm{~m})$ are the lags in the $x, y$, and $z$ directions, respectively, and $I_{x}(\mathrm{~m}), I_{y}(\mathrm{~m})$, and $I_{z}(\mathrm{~m})$ are the integral scales in the $x, y$, and $z$ directions, respectively.

\section{Field Example}

\subsection{Field Site}

[34] The site chosen to test our joint inversion method and the stochastic regularization operators is located near Eggborough, North Yorkshire, UK (Figure 1). The field site was developed to study vadose zone dynamics in Sherwood Sandstone [e.g., Binley et al., 2002a; West et al., 2003; 


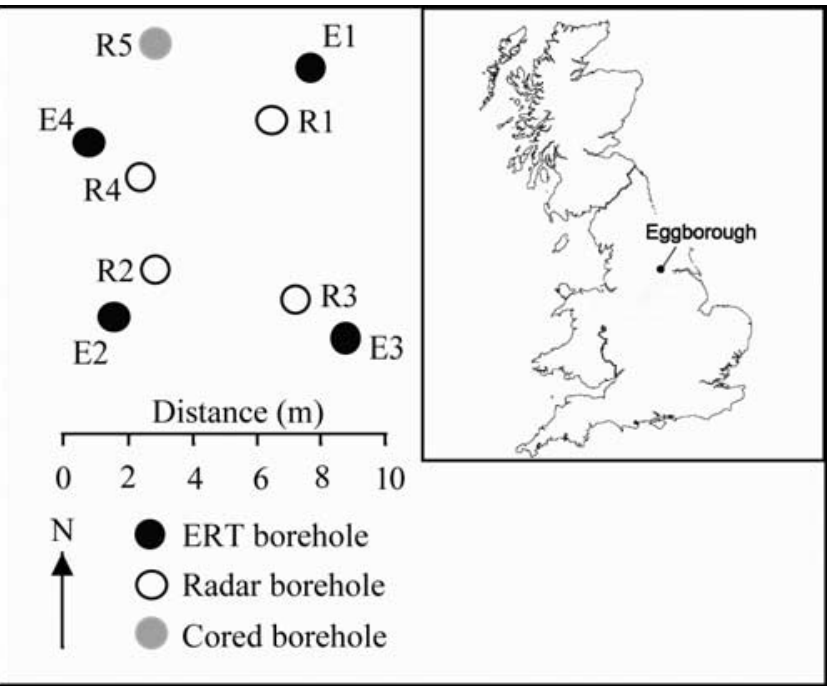

Figure 1. Areal view of the field site at Eggborough, UK $\left(53.70213^{\circ}\right.$ latitude, $-1.138707^{\circ}$ longitude).

Cassiani and Binley, 2005; Binley et al., 2005], which accounts for approximately $25 \%$ of U.K. groundwater abstraction [Allen et al., 1997]. The Sherwood Sandstone is a fluvially derived deposit consisting mainly of mediumand fine-grained sandstones with a small and variable amount of clay $(0-5 \%)$, where the fine-grained sands are thin beds 0.1 to $0.3 \mathrm{~m}$ thick, separated by much thicker (1-3 m) medium-grained units [West et al., 2003].

[35] EM conductivity logs on the transect E3-R3-R4-E4 are shown in Figure 2. A pronounced lateral layering is evident, for example, the persistent high electrical conductivity zone at $5 \mathrm{~m}$ depth and the low electrical conductivity zones at $10 \mathrm{~m}$ and $14 \mathrm{~m}$ depth. In addition to these major zones, there is also significant small-scale variability $(<1 \mathrm{~m})$.

[36] The experimental and theoretical semivariograms of the logarithm of the EM conductivity logs are shown in Figure 3. The logarithm of electrical conductivity is used because this is the parameter we invert for in the ERT inversion (see equation (1)). The semivariograms reveal a "hole effect" (i.e., the growth of the semivariogram is not monotonic but shows some weak periodicity) [e.g., Deutsch and Journel, 1992], which can be explained by the fining upward sequences in the sandstone. However, stochastic regularization operators based on the hole effect model add layers in the resulting inversion models at depths not sampled by the cross-hole geophysical data and it is therefore difficult to determine what layers that are resolved by the cross-hole geophysical data. Instead, we used a geostatistical model with a monotonic growth of the semivariogram. The stochastic regularization acts as a soft constraint (e.g., see equation (1)) and deviations from a prescribed geostatistical model is tolerated if the cross-hole geophysical data cannot be fitted otherwise. The semivariograms of the resulting tomograms might therefore reveal a hole effect, albeit damped, even if an exponential geostatistical model was used to calculate the stochastic regularization operators. We modeled the large-scale behavior with an exponential model with a variance, $c$, of 0.11 $\left(\ln (\sigma(\mathrm{S} / \mathrm{m}))^{2}\right.$ and integral scale in the vertical direction, $I_{z}$, of $3.5 \mathrm{~m}$, which are the theoretical semivariograms shown in Figure 3. Anisotropy is modeled by assuming integral scales in the horizontal directions, $I_{x}$, and $I_{y}$, to be $28 \mathrm{~m}$, i.e., eight times as long as in the horizontal directions in accordance with Cassiani and Binley [2005]. The resulting stochastic regularization operator based on this model is determined following the procedure described in section 2.6. As a comparison, we performed the ERT inversion with isotropic and anisotropic smoothing, where we penalized roughness in the horizontal directions eight times as much as in the vertical direction.

[37] West et al. [2003] fitted laboratory measurements of Sherwood Sandstone with the CRIM model [e.g., Roth et al., 1990], which is one of the most commonly used petrophysical models to interpret GPR data. They estimated that the effective relative permittivity, $\kappa_{s}$, of the minerals forming the finer lithologies of the Sherwood Sandstone is 15 at $50 \mathrm{MHz}$ compared with 5 for medium grained sandstone. However, the true relative permittivity of dry Sherwood Sandstone is between 2.7 and 4.1; and the higher effective relative permittivities were attributed to relaxation effects [West et al., 2003]. Interpretations of $100 \mathrm{MHz}$ zerooffset profiles (ZOP) in Sherwood Sandstone [Binley et al., 2004] were performed with a $\kappa_{s}$ value of 5 to estimate field moisture content. These observations are caused by the fact that the first arrival data are not affected by thin layers of (a) E3

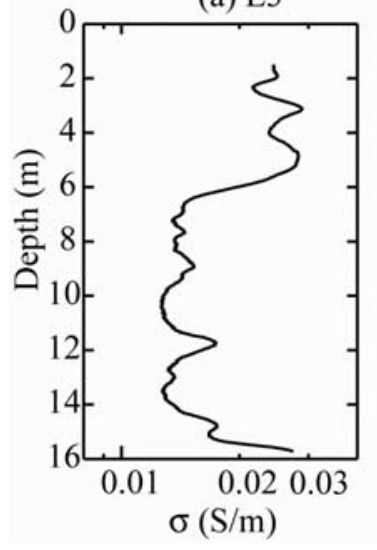

(b) R3

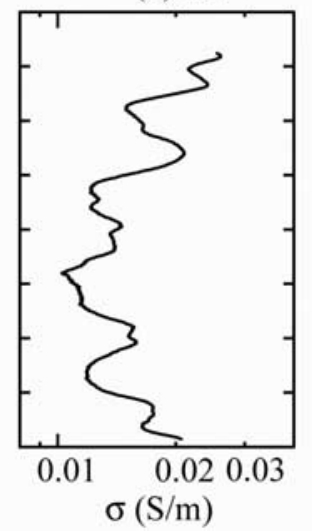

(c) R4

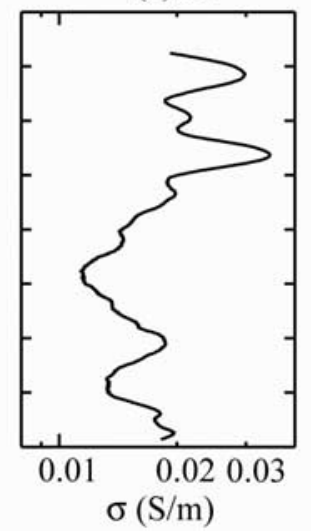

(d) E4

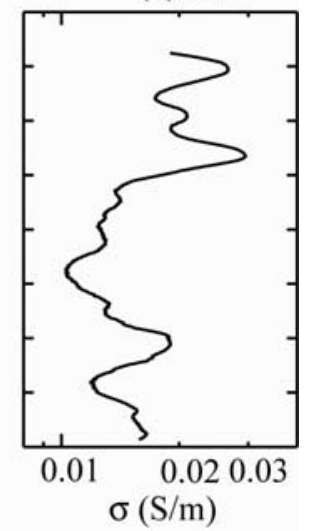

Figure 2. EM conductivity logs with $1 \mathrm{~cm}$ spacing from boreholes (a) E3, (b) R3, (c) R4, and (d) E4. 
(a) E3

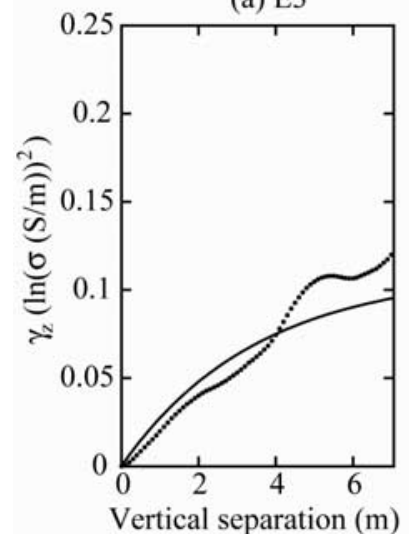

(b) R3

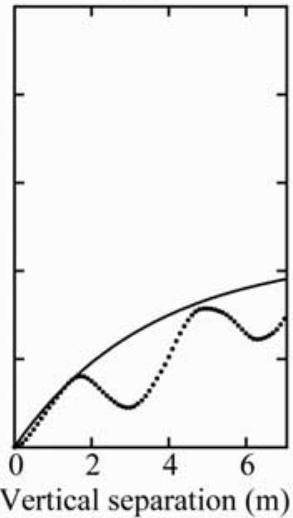

(c) R4

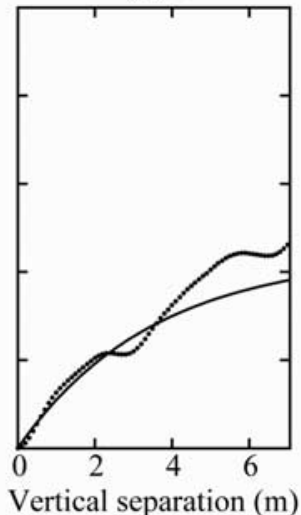

(d) E4

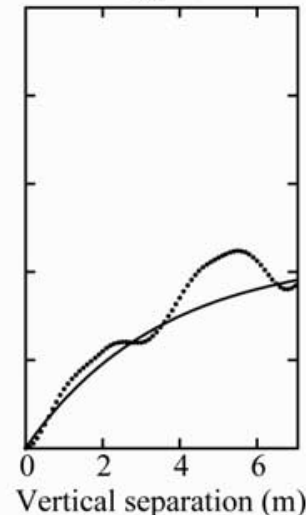

Figure 3. Semivariograms of electrical conductivity based on EM conductivity logs from boreholes (a) E3, (b) R3, (c) R4, and (d) E4, where the dotted lines indicate the experimental semivariograms and the solid lines indicate the theoretical semivariogram.

fine (slow) materials [West et al., 2003]. We decided to use the ZOP data (Figures $4 \mathrm{a}$ and $4 \mathrm{~b}$ ) to determine the vertical correlation structure and assumed the same degree of lateral anisotropy as for the ERT data, i.e., eight. The experimental and theoretical semivariograms of the ZOP profiles are shown in Figures $4 \mathrm{c}$ and $4 \mathrm{~d}$, where the theoretical semivariogram has a variance, $c$, of $0.65(\mathrm{~s} / \mathrm{m})^{2}$ and a vertical integral scale, $I_{z}$, of $3.5 \mathrm{~m}$. It should be pointed out that a)

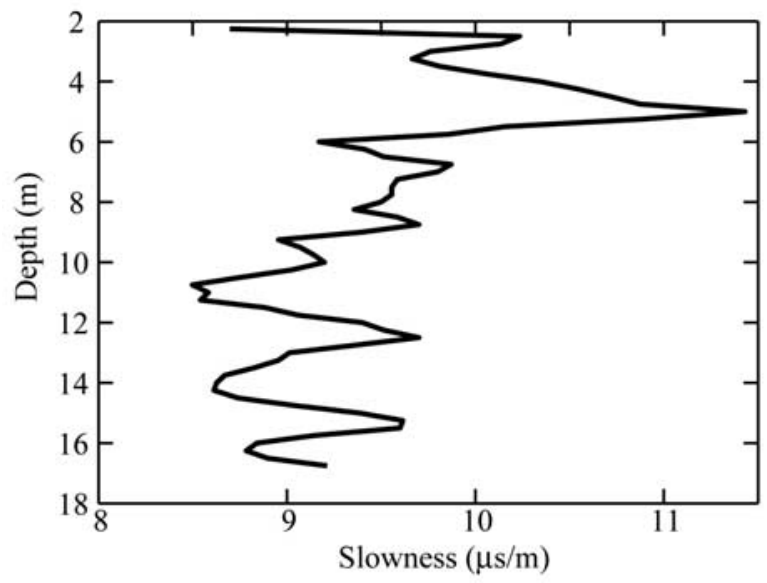

c)

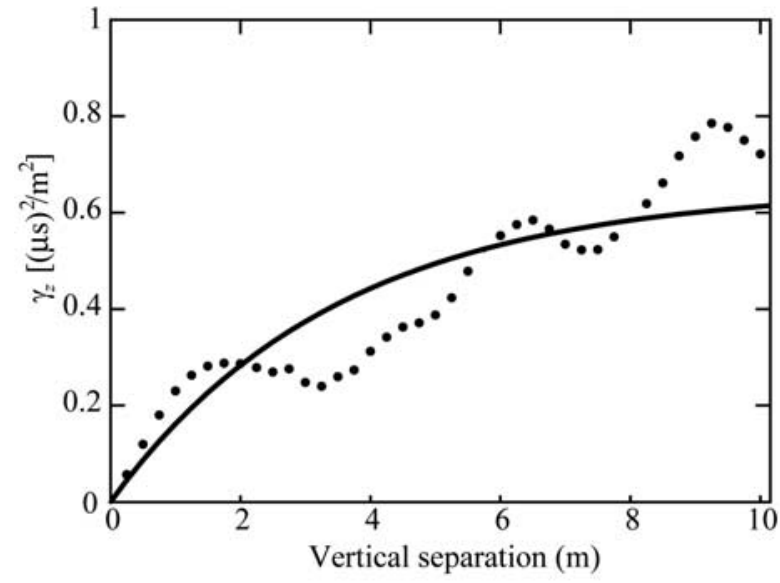

b)

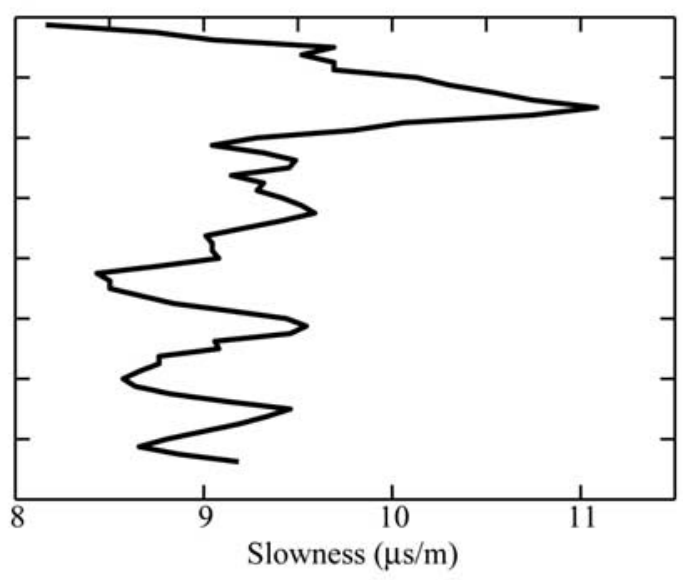

d)

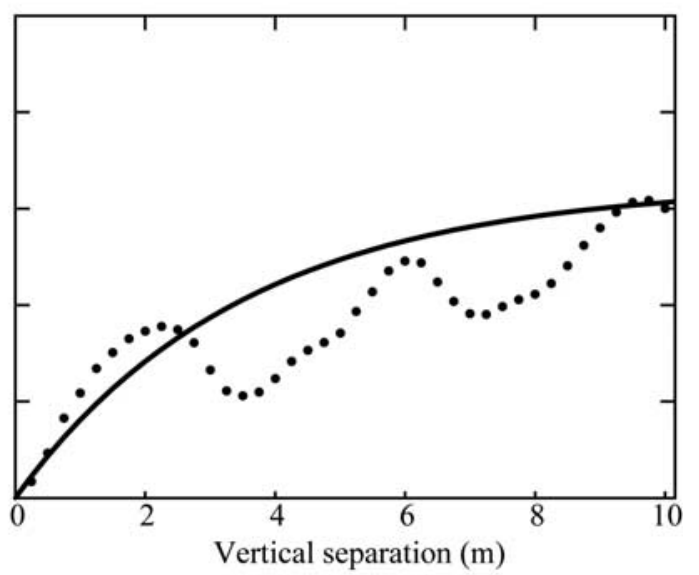

Figure 4. Inferred radar slowness from ZOP GPR data between (a) R1 and R2 and (b) R3 and R4 along with ( $c$ and $d$ ) the corresponding semivariograms, where the dotted line indicates the experimental semivariogram and the solid line indicates the theoretical semivariogram. 
these semivariograms are smoothed versions of the true variability in radar slowness mainly because of the finite frequency content of the radar signals. Nevertheless, they represent variability at a resolution that is appropriate for the inversion of the GPR traveltime data.

\subsection{ERT Inversion}

[38] The ERT data were collected in September 1999 using electrode arrays of 21 electrodes in E3 and E4 with electrode spacing of $0.75 \mathrm{~m}$. In addition, 16 surface electrodes spaced $1.1 \mathrm{~m}$ apart were used. Transfer resistances were measured in three blocks using a 64 channel multielectrode Campus Instruments Tomoplex meter. In each block of measurements, the current sink $(\mathrm{C})$ and potential reference (P) were assigned to electrodes in E3 and E4 of equal depths (4.5 m, $8.25 \mathrm{~m}$, and $12 \mathrm{~m}$ depth were used). In each block of measurements, each of the remaining 56 electrodes were used in turn as the current source $(\mathrm{C}+)$ and for each current source, the remaining 55 electrodes were used as the potential electrode $(\mathrm{P}+)$. A corresponding survey was conducted between E1 and E2.

[39] In order to decrease computing time and memory requirements, and to ensure that only high-quality data were used, we only retained the 4201 measurements for which the relative measurement errors estimated by reciprocal measurements were less than $0.7 \%$. However, error estimates based on reciprocal measurements are overoptimistic because they do not capture errors such as misplacement of electrodes or coarse model discretizations (e.g., relative errors of 5\% are possible if only two finite elements are placed between the electrodes [LaBrecque et al., 1996], which is the case in this work). It is therefore necessary to assign values to the data covariance matrix, $\mathbf{C}_{\mathrm{d}}^{\sigma}$ (equation (1)), that in addition to data errors also include estimates of modeling errors. We assumed that these errors correspond to an absolute error of $0.001 \mathrm{Ohm}$ (i.e., the accuracy of the measured transfer resistances) and a $5 \%$ relative error of the measured transfer resistances, which is a typical magnitude of data and modeling errors in crosshole ERT applications. This means that the reciprocal measurements were used to identify high-quality data, but they were not used to estimate the entries in $\mathbf{C}_{\mathrm{d}}^{\sigma}$.

[40] Forward modeling used a finite element discretization of $0.55 \mathrm{~m}$ in the horizontal directions and $0.375 \mathrm{~m}$ in the vertical direction in the area of interest, and an exponential increase in the size of the finite elements was used outside the area of interest to allow the electrical potential to decay to zero yielding a total of 46,080 finite elements. For the inverse modeling, we used 11,520 elements, which were obtained by grouping the elements in the $x$ and $y$ directions in patches of four. A uniform resistivity corresponding to the arithmetic mean of the EM conductivity logs (62 Ohm m) was used as both initial, $\mathbf{m}_{\mathrm{p}}^{\sigma}$, and a priori model, $\mathbf{m}_{\text {apriori }}^{\sigma}$.

[41] With an initial RMS of 11.4, the inversions based on the stochastic regularization (Figure 5a) and on anisotropic smoothness constraints (Figure 5b), where we penalized roughness in the horizontal directions eight times as much as in the vertical direction, reached the RMS threshold of 1 after three and five iterations, respectively. Both models have the same sequence of conductive and resistive layers, but the inversion model based on the anisotropic smoothing has a more layered structure and the conductive layers are more pronounced. Inversion with isotropic smoothing (not shown here) yielded unrealistic models (i.e., models that were not layered) but the same target data misfit.

\subsection{GPR Inversion}

[42] The MOG GPR data were collected in September 1999 with the PulseEKKO borehole radar system using $50 \mathrm{MHz}$ borehole antennae between R3 and R4 with a spacing of $0.25 \mathrm{~m}$ between $1 \mathrm{~m}$ and $16 \mathrm{~m}$ depth. A corresponding survey was conducted between R1 and R2. We restrict the inversions of the radar data to rays with angles less than $45^{\circ}$ to avoid fast raypaths within the boreholes [Peterson, 2001] and because our inversion algorithm assumes that the radar antennas act like a point source, thereby overestimating velocities when inverting data with high angular coverage [Irving and Knight, 2005]. Furthermore, we only used data that was collected below $2.0 \mathrm{~m}$ to avoid critical refraction at the soil/air interface [e.g., Rucker and Ferré, 2004] yielding 4252 first arrivals.

[43] Forward modeling was performed using a finite difference grid of $0.375 \mathrm{~m}$ in all three spatial directions yielding a total of 101,376 finite difference blocks. For the inverse modeling, we used 11,264 finite difference blocks, which were obtained by grouping the blocks in the horizontal directions in patches of nine.

[44] We assumed that the standard deviation in the data was $1.5 \mathrm{~ns}$ because this misfit yielded models with a reasonable model structure and data fit. This choice of the standard deviation is fairly subjective. Indeed, we can fit the data to a significantly lower error level, but the resolution of the resulting models are then higher than the features we can expect to resolve with $50 \mathrm{MHz}$ antennas (i.e., approximately features larger than one cubic meter). The initial slowness, $\mathbf{m}_{0}^{\mathrm{s}}$, and a priori model, $\mathbf{m}_{\text {apriori, }}^{\mathrm{s}}$, were both assumed to have a uniform slowness of $9.1 \mathrm{~ns} / \mathrm{m}$, which corresponds to the mean slowness from the ZOP GPR data (see Figures $4 \mathrm{a}$ and $4 \mathrm{~b}$ ). Both inversions converged from an initial RMS of 2.3 to the RMS threshold of 1 after four iterations.

[45] The model obtained with the stochastic regularization (Figure 5c) and the model obtained with the anisotropic smoothness constrained regularization (Figure $5 \mathrm{~d}$ ) contains the same major features: a low-velocity zone at $5 \mathrm{~m}$ depth and a high velocity zones at $10.5 \mathrm{~m}$ and $14 \mathrm{~m}$ in correspondence with the ZOPs (see Figures $4 \mathrm{a}$ and $4 \mathrm{~b}$ ). The major difference is that the model based on the anisotropic smoothing show more pronounced anomalies. Inversion with isotropic smoothing (not shown here) yielded unrealistic models (i.e., models that were not layered) but the same target data misfit.

[46] Regardless, of which type of regularization is preferred, Figure 5 illustrates nonuniqueness of the inverse problem and that inversion with different regularization operators can be useful to reveal what features that are resolved by the data and what types of features are imposed by the regularization of the inverse problem.

\subsection{Joint Cross-Hole ERT and GPR Inversion}

[47] For a given application, it is necessary to perform a few trials to find an appropriate weight to apply to the crossgradients function. The joint inversion of the data presented in sections 3.2 and 3.3 was performed using the stochastic regularization operators and $\lambda=1000$ (see equation (8)), which decreased the cross-gradients function (see equation (3)) to approximately $10 \%$ compared with the individual inver- 

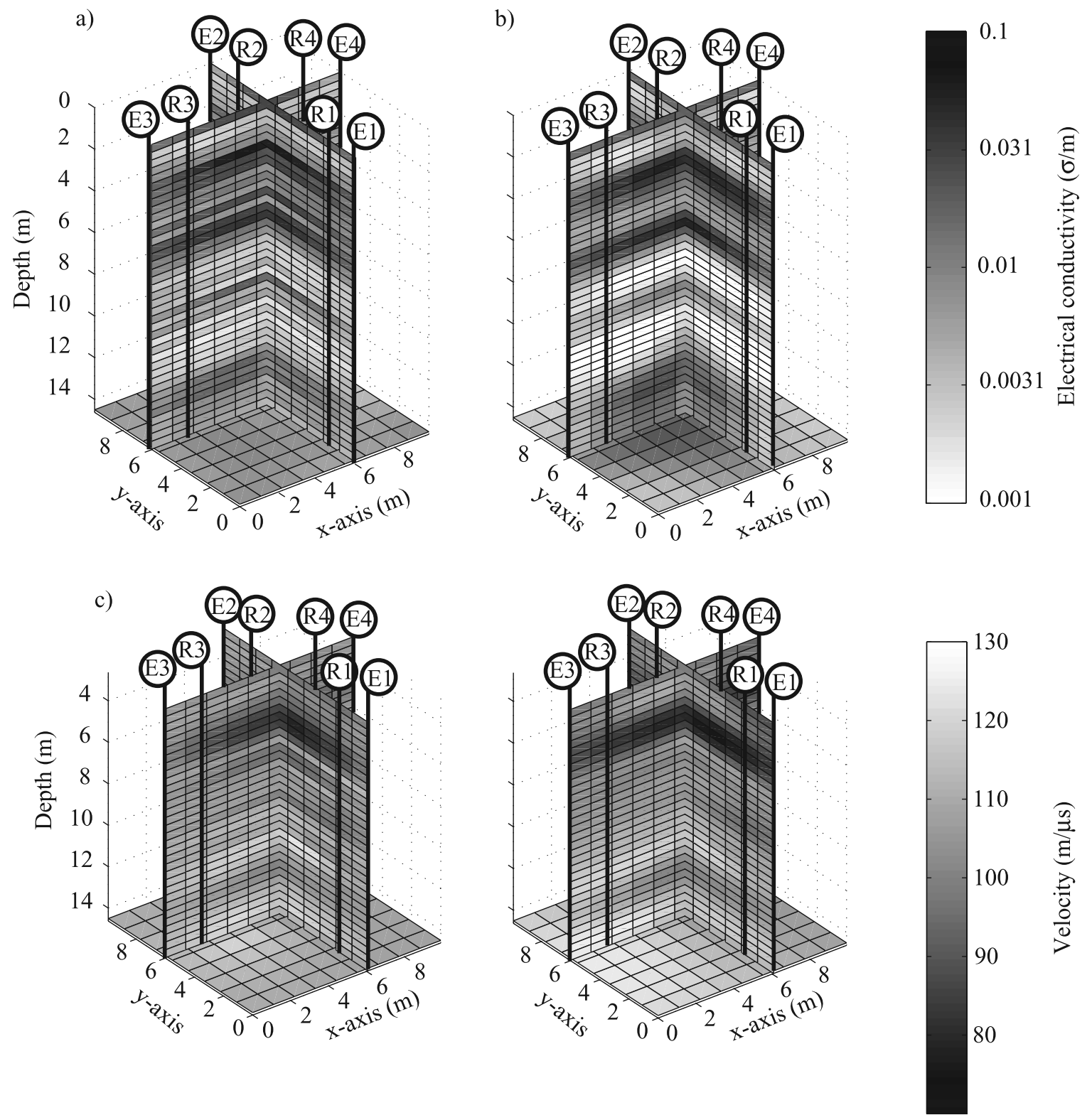

Figure 5. Individual inversion models based on (a) ERT inversion with stochastic regularization, (b) ERT inversion with anisotropic smoothness constraints, (c) MOG GPR inversion with stochastic regularization, and (d) MOG GPR inversion with anisotropic smoothness constraints.

sions. A higher value of $\lambda$ would decrease the crossgradients function further but the resulting models would be similar. The joint inversion reached the target data misfit after six iterations. The resulting resistivity model shown in Figure 6a, and the velocity model is shown in Figure 6b. The lower variability in the jointly inverted resistivity model is in accordance with the EM conductivity logs that typically show variations of a factor of three (see Figure 2). The most striking difference for the radar models are that the jointly inverted models have almost constant velocities outside the volume defined by boreholes R1, R2, R3, and R4.

\subsection{Petrophysical Implications}

[48] In this section, we derive a lithological zonation by combining the inversion results from the joint inversion (see Figure 6) with the petrophysical models (equations (10) and (11)). Scatterplots of the logarithm of electrical conductivity versus relative permittivity are first shown for the individual inversions (Figures $7 \mathrm{a}$ and $7 \mathrm{~b}$ ). These plots have no easily determined zonation patterns. Tronicke et al. [2004] illustrated that seemingly uncorrelated scatterplots of radar attenuation and radar velocity could be grouped into different zones using $k$ means clustering, a method in which pixels are iteratively regrouped into a predefined number of clusters until the variability of the values within each group is minimized.

[49] Next, we plot the corresponding scatterplots for the jointly inverted models (Figure 7c). The scatterplot reveals different slopes, where the parameters that correspond to each slope can be grouped into different zones [Gallardo and Meju, 2004; Tryggvason and Linde, 2006]. The resulting zonation can be determined without any clustering 

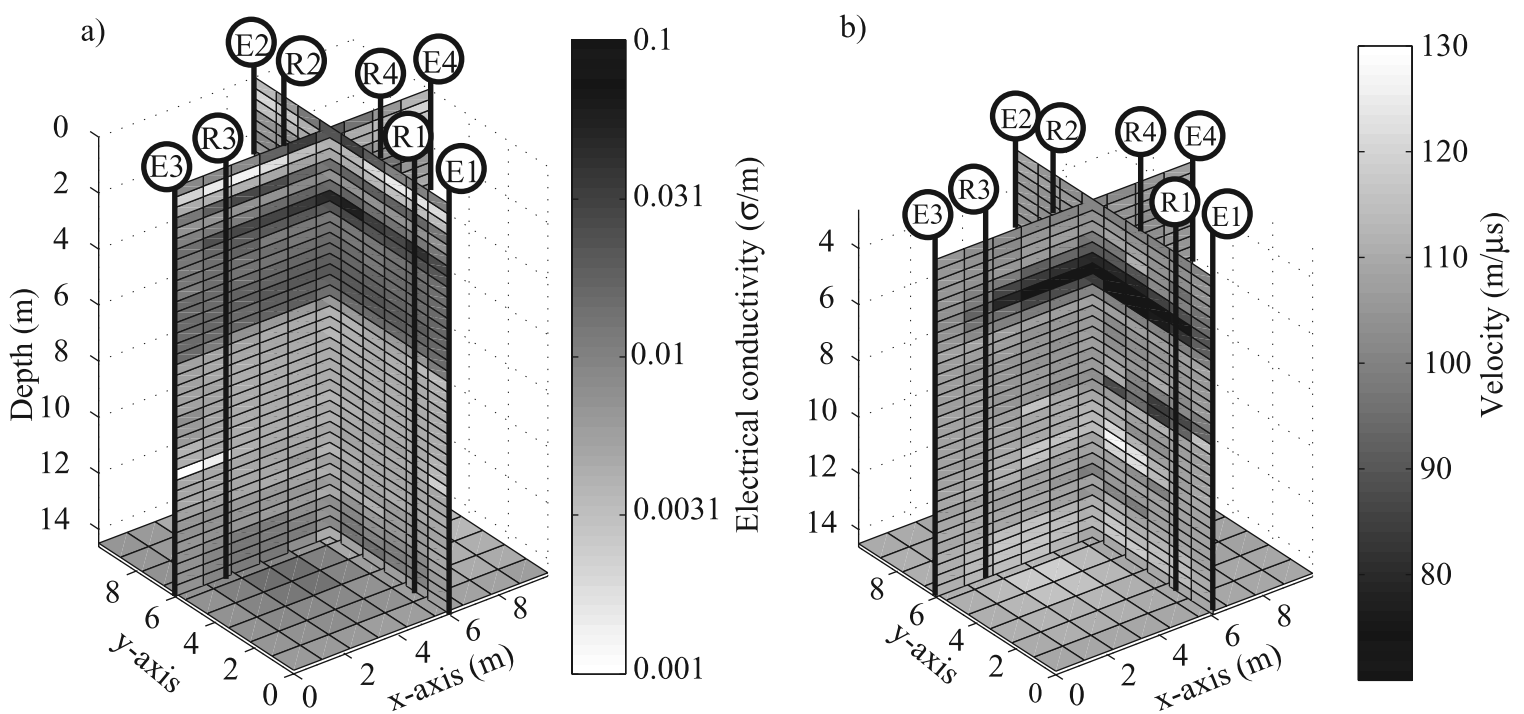

Figure 6. Joint inversion models: (a) ERT model with stochastic regularization and (b) radar velocity model with stochastic regularization.

algorithm because of the layered structure of the inverted models. A clustering algorithm must be applied if significant variations occur in more than one spatial direction. We should not interpret the apparent petrophysical relationships within each zone (i.e., the fairly straight lines corresponding to the different interpreted zones) as they represent the transitions between zones (i.e., they are strongly affected by the applied regularization). Instead, we can only hope to estimate representative values of electrical conductivity and relative permittivity for each zone.

[50] Tryggvason and Linde [2006] performed synthetic three-dimensional local earthquake tomography with joint inversion for $P$ and $S$ wave velocities by penalizing models where the cross-gradients function deviates from zero. In their synthetic example, the values of the inverted $P$ and $S$ wave velocities within their two anomalous zones corresponded to $70 \%$ of the maximum deviation from the background model. We make the assumption that these results also apply to our inversion results from Eggborough. This is a subjective choice, as we do not know if our inverse problem has the same degree of overshoot as the synthetic example presented by Tryggvason and Linde [2006]. The identified representative values are plotted as gray circles in Figure $8 \mathrm{a}$. Note that the values for the relative permittivity, $\kappa$, should be $49 \%$ of the maximum deviation as we invert for $s$ and $\kappa=c^{2} s^{2}$.

[51] The next step is to use the petrophysical models for relative permittivity (equation (10)) and electrical conductivity (equation (11)) to infer possible values of the rock properties and state variables of the identified lithological zones. We defined typical ranges for weakly consolidated sandstones and siltstones for the parameters that enter these petrophysical models according to $\phi=\mathrm{U}[0.25-$ 0.35] [Binley et al., 2005], $S_{w}=\mathrm{U}[0,1.0], n=\mathrm{U}[1.3,2.2]$, $m=\mathrm{U}[1.3,2.2]$ [Revil et al., 1998], the logarithm of $R_{\text {eff }}$ is $\mathrm{U}[-7.2,-5], \sigma_{w}=0.10 \mathrm{~S} / \mathrm{m}$ [Binley et al., 2005], $\kappa_{w}=81$, a)

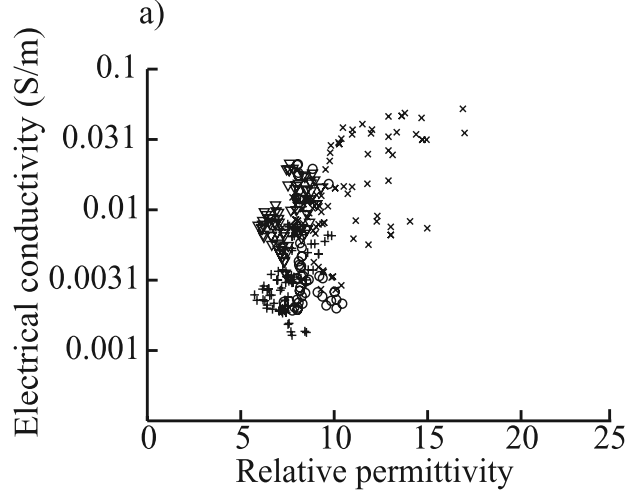

b)

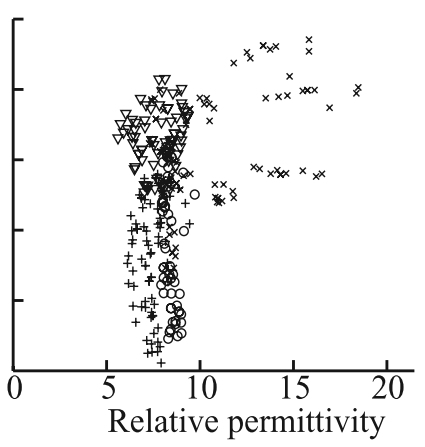

c)

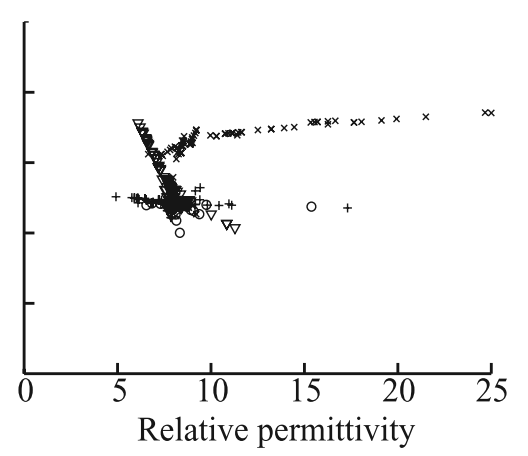

Figure 7. Scatterplots of $\log 10$ of electrical conductivity versus relative permittivity, shown for the model parameters that correspond to the transects between boreholes R1 and R2 and R3 and R4 for the individually inverted models based on (a) the stochastic regularization and (b) the anisotropic smoothness constraints and for the jointly inverted models based on (c) the stochastic regularization. The zonation is based on the trends in the scatterplots of the models based on the joint inversion with stochastic regularization, where the crosses, circles, pluses, and triangles indicate zones 1-4, respectively. 


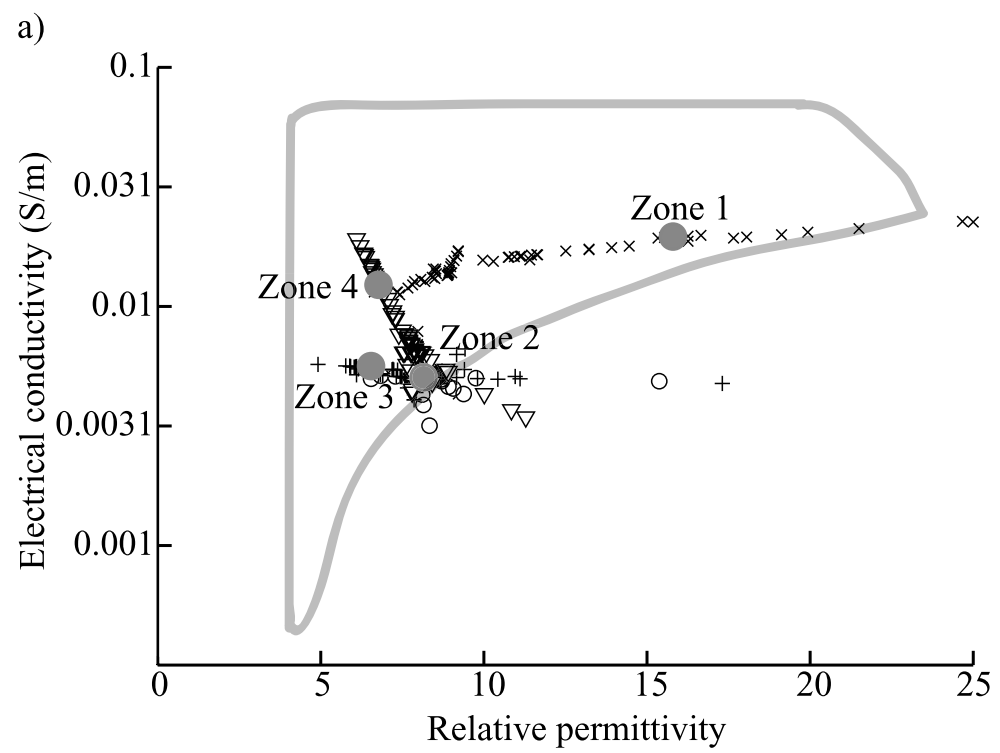

b)

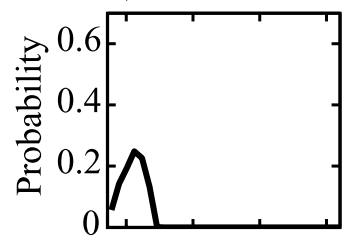

g)

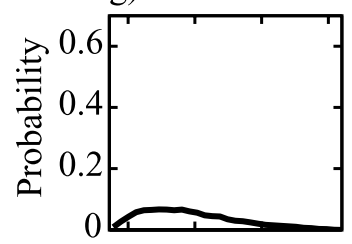

1)

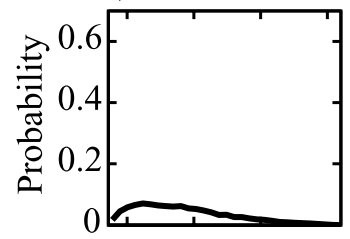

q)

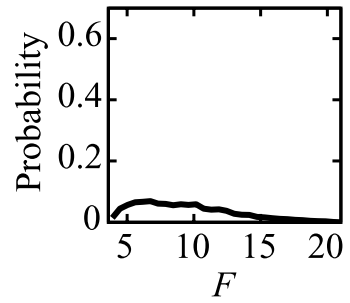

c)

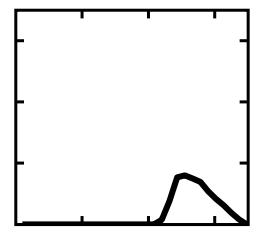

h)

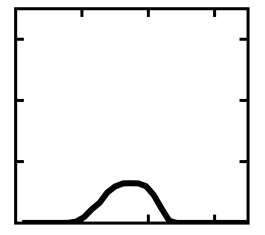

$\mathrm{m})$

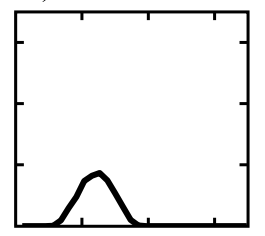

r)

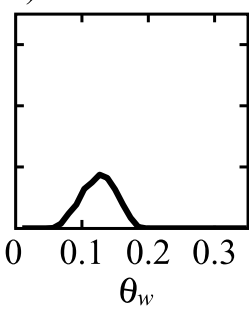

d)

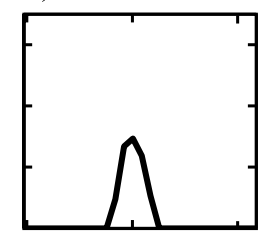

i)

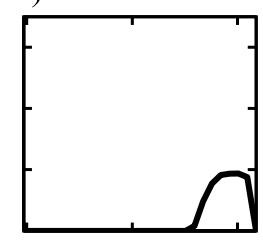

n)

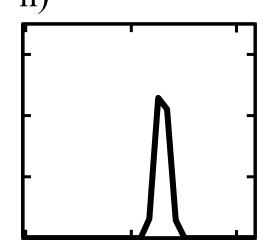

s)

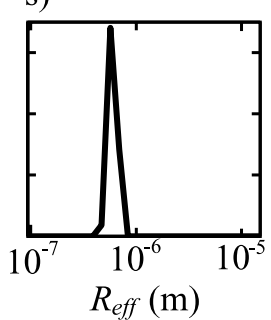

e)

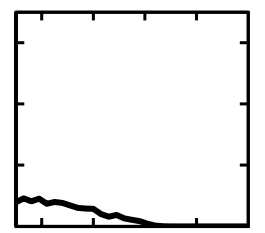

j)

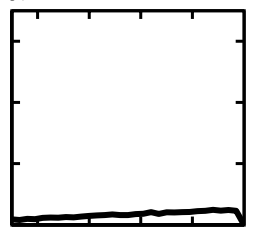

o)

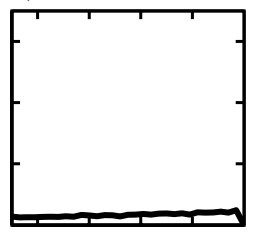

t)

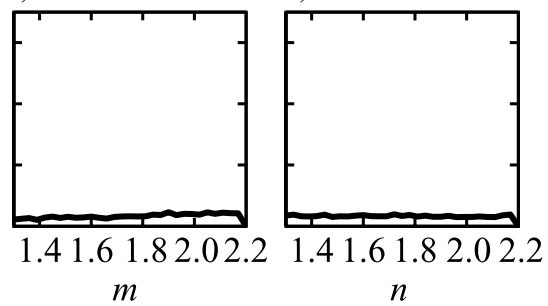

Figure 8. (a) Four points in the scatterplots based on the jointly inverted models with stochastic regularization, marked with gray circles. These points are assumed to be representative of four different lithological zones. The solid gray line indicates the range of possible relationships based on equations (10) $-(12)$ and the parameter ranges specified in section 3.5. Probability density functions of (b) $F$, (c) $\theta$, (d) $R_{\text {eff }}$, (e) $m$, and (f) $n$ are shown for zone 1 . Corresponding plots are shown for $(\mathrm{g}-\mathrm{k})$ zone $2,(1-\mathrm{p})$ zone 3 , and $(\mathrm{q}-\mathrm{u})$ zone 4. 
$\kappa_{s}=5$ [Binley et al., 2004], $\kappa_{a}=1$ and $\Sigma_{s}=2.5 \times 10^{-9} \mathrm{~S}$ [Revil and Glover, 1998], where U[·] denotes a uniform distribution. The resulting range for $F=\phi^{-m}$ is 3.9 to 21 . It is then possible to calculate the bounds of the possible relationships between $\sigma_{\text {eff }}$ and $\kappa_{\text {eff }}$ by generating a large set of random realizations from these distributions and computing the resulting values of $\sigma_{\text {eff }}$ and $\kappa_{\text {eff. }}$. The resulting allowable ranges of $\sigma_{\text {eff }}$ and $\kappa_{\text {eff }}$ are given by the gray solid line in Figure 8a. If we assume that the petrophysical models (equations (10) and (11)) are reasonably accurate, it is possible to identify areas of the model where the inversion results indicate unphysical relationships caused by the inversion process and measurement errors. We see that the values of the scatterplot in Figure 8a mostly fall within the allowable ranges.

[52] Next we plotted the probability density functions of some of the parameters entering equations (10)-(12) for the identified zones (Figures $8 \mathrm{~b}-8 \mathrm{u}$ ). This was achieved by drawing a large number of realizations (i.e., several millions) from the uniform distributions identified above and storing all realizations where the resulting $\sigma_{\text {eff }}$ and $\kappa_{\text {eff }}$ are close to the values specified for the different zones (i.e., the gray circles in Figure 8a). Parameters that have close to a uniform distribution are poorly defined, whereas parameters with a well-defined peak are well defined by the joint inversion. For example, both $\phi$ and $m$ are poorly defined, but the resulting $F=\phi^{-m}$ have fairly well defined peaks between 4.5 and 6 (see for example Figure 8b). Also, the water content $\theta_{w}=S_{w} \phi$ is well defined (see for example Figure 8c). It is not possible to resolve $\sigma_{s}$ using only ERT or GPR traveltimes. However, if ERT and GPR traveltimes are combined, $\sigma_{s}$ and thus $R$ (see equation (12)) are welldefined parameters (see for example Figure 8d) at low $S_{w}$ when the relative contribution of $\sigma_{s}$ to $\sigma_{\text {eff }}$ is the largest (see equation (11)).

[53] Six core samples from a neighboring borehole core (see R5 in Figure 1) were analyzed for the electrical formation factor, $F$, and the values ranged between 2.6 and 5 [Binley et al., 2005]. The correspondence with the estimated formation factors from the joint inversion (see Figures $8 \mathrm{~b}, 8 \mathrm{~g}, 81$, and $8 \mathrm{q}$ ) is acceptable, keeping in mind that the laboratory measurements, $F^{a p p}=\sigma_{w} / \sigma_{\text {eff }}$, represent apparent electrical formation factors at $\sigma_{w}=0.10 \mathrm{~S} / \mathrm{m}$ as $\sigma_{s}$ is neglected, thereby underestimating $F$ when $\sigma_{s}$ is significant. Furthermore, any disturbance during coring decreases the measured $F$ in the cores in comparison with the intact formation.

[54] Binley et al. [2004] estimated that water content varies in the range 0.08 and 0.18 between boreholes R3 and R4 using GPR zero-offset profiles and assuming $\kappa_{s}=5$. These estimates represent averaged volumes with a thickness of approximately $1 \mathrm{~m}$ and local variations in $\theta_{w}$ are likely to be larger. Indeed, high-resolution time domain reflectometry surveying at a neighboring borehole performed during one year suggest that $\theta_{w}$ at some depth intervals ranged between 0.15 to 0.33 [West and Truss, 2006]. The $\theta_{w}$ with the maximum likelihood in each zone are $0.26,0.19,0.13$, and 0.12 , respectively. These estimates honor the lower bounds suggested by Binley et al. [2004]. The estimated water contents are likely to be biased downward because the GPR waves travel preferably along the fast zones with low saturation and the slow zones with high saturation are therefore poorly sampled and poorly constrained in the inversion. The estimated ranges of $\theta_{w}$ (see Figures $8 \mathrm{c} 8 \mathrm{~h}, 8 \mathrm{~m}$, and $8 \mathrm{r}$ ) could be reduced if $m$ and $n$ could be constrained within a smaller range.

[55] The number of gamma ray counts is often assumed to be a linear function of clay content [e.g., Revil et al., 1998] and thus $\sigma_{s}$ if the mineralogy of the clay fraction is fairly constant. The clay fraction in the cored borehole (Figure 9b) defined as the fraction of the sample with a grain size smaller than $2.01 \mu \mathrm{m}$ and gamma ray logs (Figure 9c) conducted in the boreholes [Binley et al., 2004] were therefore used to evaluate if the differences in $\sigma_{s}$ for zones 1 and 4 are reasonable. The highest gamma ray count for all boreholes in the depth range of zone 1 corresponds to a small anomaly with a peak of $90 \mathrm{cps}$, whereas the highest gamma ray count in zone 4 corresponds to a thicker anomaly with a peak of $133 \mathrm{cps}$ (see also Figure 9c). At least qualitatively, this indicates that surface conduction effects are more pronounced in zone 4 . These results indicate that we can estimate $F, \theta_{w}$, and $R_{\text {eff }}$ reasonably well by jointly inverting ERT and GPR traveltime data in unsaturated sandstone.

[56] Inversion results have resolution limitations that can be quite significant when attempting quantitative interpretations [e.g., Day-Lewis and Lane, 2004; Day-Lewis et al., 2005], but these aspects of the inversion problem are outside the scope of this study. Furthermore, the petrophysical models (equations (10) and (11)) are simple and do not capture all variability in $\sigma_{\text {eff }}$ and $\kappa_{\text {eff. }}$. Consequently, the values of the parameters of equations (10) and (11) shown in Figure 8 should be considered as rough estimates, but we believe that the relative differences between the zones are well resolved.

[57] The zonation derived at Eggborough provides estimates of the geometry and the associated properties of four different zones, but we cannot expect to resolve variations within each zone. To understand vadose zone dynamics it is necessary to complement cross-hole methods with measurements of higher resolution. West and Truss [2006] studied temporal and spatial variations in soil moisture at a neighboring site using time domain reflectometry. They found that there is significant lateral flow bypassing less permeable layers. Preferential flow paths of a few decimeters thickness or thinner cannot be resolved using cross-hole ERT and $50 \mathrm{MHz}$ GPR radar data with the borehole spacing used at Eggborough.

\section{Discussion and Conclusions}

[58] In hydrogeophysics, geophysical information is often used to constrain or produce hydrogeological models [e.g., Hubbard et al., 2001; Cassiani and Binley, 2005]. Therefore it is important that geophysical models are consistent with available data and the modeler's perception of the hydrogeological system, as well as the parameterization used in the subsequent hydrogeological modeling. Regularization operators in geophysical inverse problems should be chosen with care because they have, for a given data misfit, a significant influence on the resulting models; this is the case even for joint inversion of high-resolution ERT and MOG GPR traveltime data (see Figure 6). It can be useful to perform several inversions with different regularization operators (i.e., different geostatistical model types and 
a)

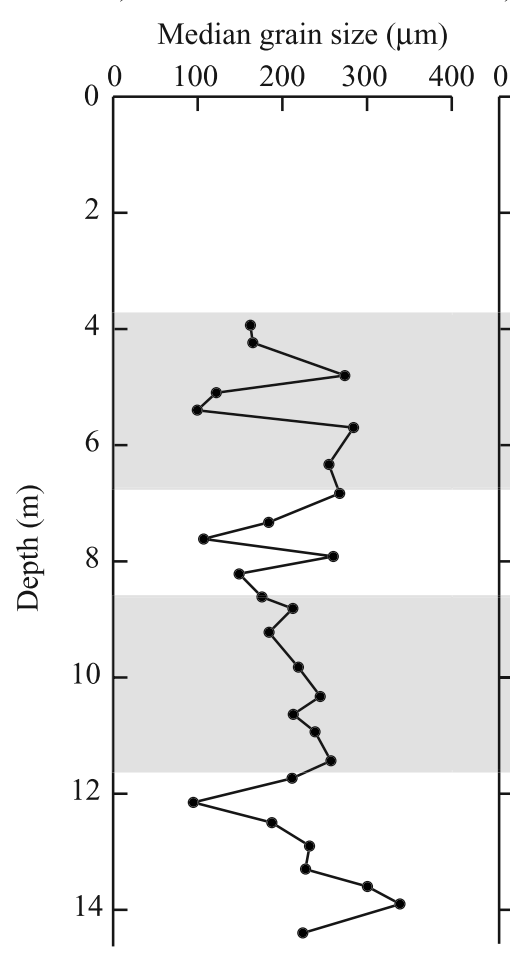

b)

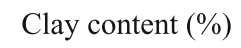

c)

Gamma (cps)

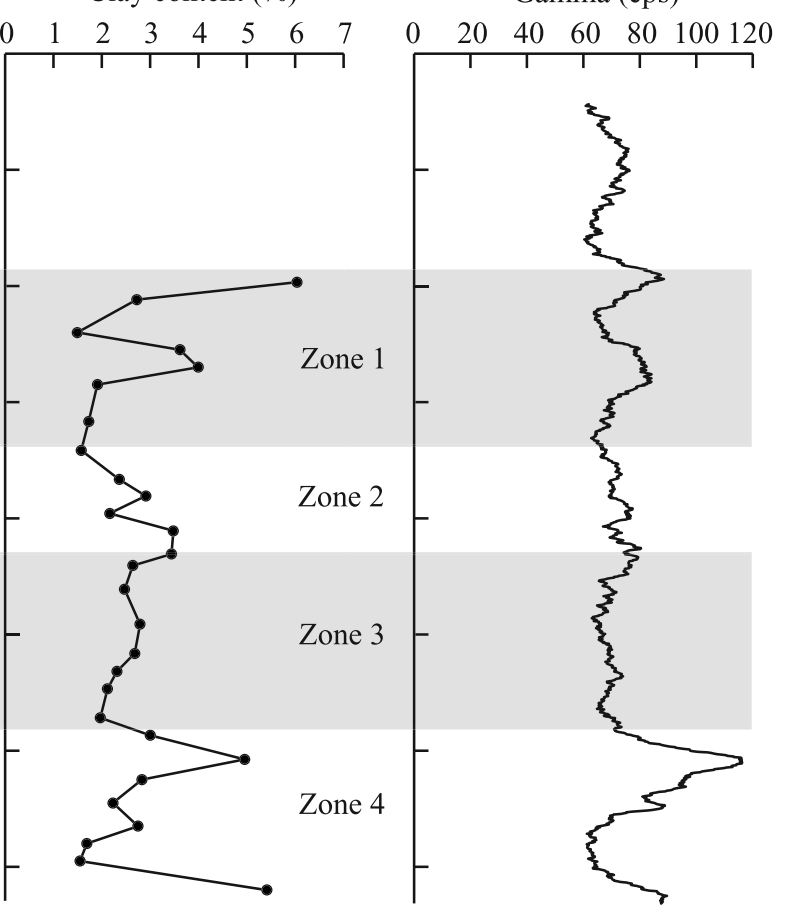

Figure 9. (a) Median particle size and (b) clay fraction in cored borehole (R5) together with (c) the natural gamma log from the neighboring borehole E4. The zonation based on joint inversion of ERT and MOG GPR traveltime data with stochastic regularization operators is shown in the background.

different choices of their parameters) to assess what features are resolved by the data. In this work, we introduced an efficient way to calculate stochastic regularization operators based on geostatistical models estimated from EM conductivity $\operatorname{logs}$ and GPR ZOPs. It should be noted that the stochastic regularization operators used in this work are affected by both the underlying geology and the water content (see section 2.5). In applications to the saturated zone, the stochastic regularization operators would correspond to the underlying geology only.

[59] There is no apparent correlation between the individually inverted ERT and MOG GPR tomograms from Eggborough (see Figures $7 \mathrm{a}$ and $7 \mathrm{~b}$ ) making joint inversion with a functional relationship infeasible. Instead, we constrained the values of the cross-gradients function (see equation (3)), which quantifies deviations from structural similarity, where models are defined as structurally similar if the gradients of the models have the same or opposite direction [Gallardo and Meju, 2003].

[60] A structural approach to joint inversion, together with stochastic regularization operators, made it possible to jointly invert different data sets and obtain geologically realistic models at a research site that was selected to study vadose zone dynamics in Sherwood Sandstone without imposing overly restrictive assumptions. The threedimensional joint inversion of cross-hole ERT and MOG GPR traveltime data also allowed a lithological zonation of the site. This zonation was not apparent from the individually inverted models. Furthermore, the joint inversion made it possible to estimate probability density functions of the electrical formation factor, $F$, the water content, $\theta_{w}$, and the effective grain radius of the sediments, $R_{\text {eff, }}$ in the different zones. Core measurements and gamma logs were in qualitative agreement with the derived properties of the different zones.

[61] The scatterplots of the jointly inverted models (Figure 8a) have different slopes for different zones. These slopes should not be considered to represent the intrinsic petrophysical relationship between electrical conductivity and relative permittivity within each zone, as these slopes are strongly influenced by both the regularization used to solve the inverse problem and the resolution limitations of the geophysical methods used. Our three-dimensional joint inversion of cross-hole ERT and GPR traveltime data allowed us to estimate the bulk properties of each zone, but it did not allow us to resolve small-scale variability within the zones.

\section{Appendix A: Effective Relative Permittivity}

[62] Pride [1994] used a volume-averaging approach to derive the following expression for the effective relative permittivity, $\kappa_{\text {eff, }}$ of two-phase media (see his equation (253))

$$
\kappa_{\text {eff }}=\left[\frac{\phi}{\alpha}\left(\kappa_{w}-\kappa_{s}\right)+\kappa_{s}\right],
$$

where $\phi$ is the porosity, $\alpha$ is the tortuosity, $\kappa_{w}$ and $\kappa_{s}$ are the relative permittivities of the saturated pore space and the rock matrix, respectively. If the pore space is a mixture of two immiscible fluids (here water and air), we can apply a 
similar methodology to derive the effective permittivity of this mixture, $\kappa_{\phi}$. This yields:

$$
\kappa_{\phi}=\left[\frac{S_{w}}{\alpha_{w}}\left(\kappa_{w}-\kappa_{a}\right)+\kappa_{a}\right]
$$

where $\alpha_{w}$ is the tortuosity of the water phase relative to the mixture, $S_{w}$ is the water saturation, and $\kappa_{a}$ is the relative permittivity of air. To be compatible with Archie's first and second laws, we must have

$$
F=\frac{\alpha_{w}}{\phi}
$$

where $F$ is the electrical formation factor and

$$
S^{-n}=\frac{\alpha_{w}}{S_{w}}
$$

where $n$ is Archie's second exponent. By inserting equation (A2) into equation (A1) and using equations (A3) and (A4), we arrive at the following expression for the effective relative permittivity of partially saturated porous media

$$
\kappa_{\text {eff }}=\frac{1}{F}\left[S_{w}^{n} \kappa_{w}+\left(1-S_{w}^{n}\right) \kappa_{a}+(F-1) \kappa_{s}\right]
$$

[63] The boundary value problem for the electrical conductivity is similar to that of relative permittivity [Pride, 1994], except that the gas phase is insulating (i.e., $\sigma_{a}=0$ ). Despite the fact that the grains are insulating, the grains are coated by the electrical double layer [e.g., Leroy and Revil, 2004], which gives them an equivalent grain conductivity.

[64] Acknowledgments. This research was supported, in part, by the Swedish Geological Survey under contract 60-1307/2004 awarded to Niklas Linde. Field data were obtained through funding from the UK Natural Environment Research Council (NERC) grant GR3/1150, and core analysis was funded under the UK NERC grant NER/A/S/2001/01175 (both awarded to Andrew Binley). Peter Winship (Lancaster University) collected the field data and spent many hours picking first arrivals. The application here would not have been possible without his support. We thank Associate Editor Lee Slater and the three anonymous reviewers for constructive reviews that helped to improve the clarity of the paper.

\section{References}

Allen, D. L., L. J. Brewerton, L. M. Coleby, B. R. Gibbes. M. A. Lewis, A. M. MacDonald, S. J. Wagstaff, and A. T. Williams (1997), The physical properties of major aquifers in England and Wales, Tech. Rep. WD/95/34, 312 pp., Br. Geol. Surv., Keyworth, U. K.

Binley, A. M. (2005), R3-Three-dimensional inversion of electrical resistance tomography data, report, Lancaster Univ., Lancaster, U. K.

Binley, A., G. Cassiani, R. Middleton, and P. Winship (2002a), Vadose zone flow model parameterisation using cross-borehole radar and resistivity imaging, J. Hydrol., 267(3-4), 147-159.

Binley, A., P. Winship, L. J. West, M. Pokar, and R. Middleton (2002b), Seasonal variation of moisture content in unsaturated sandstone inferred from borehole radar and resistivity profiles, J. Hydrol., 267(3-4), 160-172.

Binley, A., G. Cassiani, and P. Winship (2004), Characterization of heterogeneity in unsaturated sandstone using borehole logs and cross-borehole tomography, in Aquifer Characterization, edited by J. S. Bridge, and D. W. Hyndman, Spec. Publ. SEPM Soc. Sediment. Geol., 80, 129-138.

Binley, A., L. D. Slater, M. Fukes, and G. Cassiani (2005), Relationship between spectral induced polarization and hydraulic properties of saturated and unsaturated sandstone, Water Resour. Res., 41, W12417, doi:10.1029/2005WR004202.
Cassiani, G., and A. Binley (2005), Modeling unsaturated flow in a layered formation under quasi-steady state conditions using geophysical data constraints, Adv. Water Resour., 28(5), 467-477.

Constable, S. C., R. L. Parker, and C. G. Constable (1987), Occam's inversion-A practical algorithm for generating smooth models from electromagnetic sounding data, Geophysics, 52(3), 289-300.

Day-Lewis, F. D., and J. W. Lane, Jr. (2004), Assessing the resolutiondependent utility of tomograms for geostatistics, Geophys. Res. Lett., 31, L07503, doi:10.1029/2004GL019617.

Day-Lewis, F. D., K. Singha, and A. M. Binley (2005), applying petrophysical models to radar travel time and electrical resistivity tomograms: Resolution-dependent limitations, J. Geophys. Res., 110, B08206, doi:10.1029/2004JB003569.

Deutsch, C., and A. Journel (1992), GSLIB: A Geostatistical Software Library and User's Guide, 2nd ed., Oxford Univ. Press, New York.

Dietrich, C. R., and G. N. Newsam (1997), Fast and exact simulation of stationary Gaussian processes through circulant embedding of the covariance matrix, SIAM J. Sci. Comput., 18(4), 1088-1107.

Gallardo, L. A., and M. A. Meju (2003), Characterization of heterogeneous near-surface materials by joint $2 \mathrm{D}$ inversion of dc resistivity and seismic data, Geophys. Res. Lett., 30(13), 1658, doi:10.1029/2003GL017370.

Gallardo, L. A., and M. A. Meju (2004), Joint two-dimensional DC resistivity and seismic travel time inversion with cross-gradient constraints, J. Geophys. Res., 109, B03311, doi:10.1029/2003JB002716.

Geselowitz, D. B. (1971), An application of electrocardiographic lead theory to impedance plethysmography, IEEE Trans. Biomed. Eng., 18, $38-41$.

Golub, G. H., and C. F. Van Loan (1996), Matrix Computations, 3rd ed., Johns Hopkins Univ. Press, Baltimore, Md.

Haber, E., and D. Oldenburg (1997), Joint inversion: A structural approach, Inverse Probl., 13(1), 63-77.

Hole, J. A. (1992), Nonlinear high-resolution three-dimensional seismic travel time tomography, J. Geophys Res., 97(B5), 6553-6562.

Hubbard, S. S., J. Chen, J. Peterson, E. L. Mayer, K. H. Williams, D. J. Swift, B. Mailloux, and Y. Rubin (2001), Hydrogeological characterization of the South Oyster Bacterial Transport Site using geophysical data, Water Resour. Res., 37(10), 2431-2456.

Irving, J. D., and R. Knight (2005), Effect of antennas on velocity estimates obtained from crosshole GPR data, Geophysics, 70(5), K39-K42, doi:10.1190/1.2049349.

Kitanidis, P. K., and E. G. Vomvoris (1983), A geostatistical approach to the inverse problem in groundwater modeling (steady-state) and onedimensional simulations, Water Resour. Res., 19(3), 677-690.

LaBrecque, D. J., M. Miletto, W. Daily, A. Ramirez, and E. Owen (1996), The effects of noise on Occam's inversion of resistivity tomography data, Geophysics, 61(2), 538-548.

Leroy, P., and A. Revil (2004), A triple layer model of the surface electrochemical properties of clay minerals, J. Colloid Interf. Sci., 270(2), 371-380.

Linde, N., S. Finterle, and S. Hubbard (2006), Inversion of tracer test data using tomographic constraints, Water Resour. Res., 42, W04410, doi:10.1029/2004WR003806.

Lines, L. R., A. K. Schultz, and S. Treitel (1988), Cooperative inversion of geophysical data, Geophysics, 53(1), 8-20.

Maurer, H., K. Holliger, and D. E. Boerner (1998), Stochastic regularization: Smoothness or similarity?, Geophys. Res. Lett., 25(15), $2889-2892$.

Marquardt, D. W. (1970), Generalized inverses, ridge regression, biased linear estimation, and non-linear estimation, Technometrics, 12, $591-612$.

Mavko, G., T. Mukerji, and J. Dvorkin (1998), The Rock Physics Handbook-Tools for Seismic Analysis of Porous Media, Cambridge Univ. Press, New York.

Menke, W. (1984), Geophysical Data Analysis: Discrete Inverse Theory, Elsevier, New York.

Mosegaard, K., and A. Tarantola (1995), Monte-Carlo sampling of solutions to inverse problems, J. Geophys. Res., 100(B7), 12,431-12,447.

Musil, M., H. R. Maurer, and A. G. Green (2003), Discrete tomography and joint inversion for loosely connected or unconnected physical properties: Application to crosshole seismic and georadar data sets, Geophys. J. Int., $153(2), 389-402$.

Nowak, W., S. Tenkleve, and O. A. Cirpka (2003), Efficient computation of linearized cross-covariance and auto-covariance matrices of interdependent quantities, Math. Geol., 35(1), 53-66.

Paige, C. C., and M. A. Saunders (1982), LSQR: An algorithm for sparse linear equations and sparse least squares, Trans. Math. Software, 8(1), $43-71$. 
Parker, R. L. (1994), Geophysical Inverse Theory, Princeton Univ. Press, Princeton, N. J.

Peterson, J. E. Jr. (2001), Pre-inversion processing and analysis of tomographic radar data, J. Environ. Eng. Geophys., 6(1), 1-18.

Podvin, P., and I. Lecomte (1991), Finite difference computation of travel times in very contrasted velocity models: A massively parallel approach and its associated tools, Geophys. J. Int., 105(1), 271-284.

Pride, S. (1994), Governing equations for the coupled electromagnetics and acoustics of porous media, Phys. Rev. B, 50(21), 15,678-15,696.

Ranguelova, E. B. (2002), Segmentation of textured images on threedimensional lattices, Ph.D. dissertation, Univ. of Dublin, Dublin.

Revil, A., and L. M. Cathles (1999), Permeability of shaly sands, Water Resour. Res., 35(3), 651-662.

Revil, A., and P. J. Glover (1998), Nature of surface electrical conductivity in natural sands, sandstones, and clays, Geophys. Res. Lett., 25(5), 691-694.

Revil, A., L. M. Cathles, S. Losh, and J. A. Nunn (1998), Electrical conductivity in shaly sands with geophysical applications, J. Geophys. Res., 103(B10), 23,925-23,936.

Roth, K. R., R. Schulin, H. Fluhler, and W. Attinger (1990), Calibration of time domain reflectometry for water content measurement using a composite dielectric approach, Water Resour. Res., 26(10), 2267-2273.

Rucker, D. F., and T. P. A. Ferré (2004), Near-surface water content estimation with borehole ground penetrating radar using critically refracted waves, Vadose Zone J., 3, 278-287.

Schön, J. H. (1996), Physical Properties of Rocks-Fundamentals and Principles of Petrophysics, Elsevier, New York.

Siripunvaraporn, W., and G. Egbert (2000), An efficient data-subspace inversion method for 2-D magnetotelluric data, Geophysics, 65(3), $791-803$.

Tikhonov, A. N., and V. Y. Arsenin (1977), Solutions of Ill-Posed Problems, John Wiley, Hoboken, N. J.

Tronicke, J., K. Holliger, W. Barrash, and M. D. Knoll (2004), Multivariate analysis of cross-hole georadar velocity and attenuation tomograms for aquifer zonation, Water Resour. Res., 40, W01519, doi:10.1029/ 2003WR002031.
Tryggvason, A., and N. Linde (2006), Local earthquake (LE) tomography with joint inversion for P- and S-wave velocities using structural constraints, Geophys. Res. Lett., 33, L07303, doi:10.1029/2005GL025485.

Tryggvason, A., S. T. Rögnvaldsson, and Ó. G. Flovenz (2002), Threedimensional imaging of the P- and S-wave velocity structure and earthquake locations beneath southwest Iceland, Geophys. J. Int., 151(3), $848-866$.

Vozoff, K., and D. L. B. Jupp (1975), Joint inversion of geophysical data, Geophys. J.R. Astron. Soc., 42, 977-991.

Waxman, M. H., and L. J. M. Smits (1968), Electrical conductivities in oilbearing shaly sands, Soc. Pet. Eng. J., 8, 107-122.

West, L. J., and S. W. Truss (2006), Borehole time domain reflectometry in layered sandstone: Impact of measurement technique on vadose zone process identification, J. Hydrol., 319(1-4), 143-162.

West, L. J., K. Handley, Y. Huang, and M. Pokar (2003), Radar frequency dielectric dispersion in sandstone: Implications for determination of moisture and clay content, Water Resour. Res., 39(2), 1026, doi:10.1029/2001WR000923.

Yeh, T-.C. J., M. Jin, and S. Hanna (1996), An iterative stochastic inverse method: Conditional effective transmissivity and hydraulic head fields, Water Resour. Res., 32(1), 85-92.

Yeh, T-.C. J., S. Liu, R. J. Glass, K. Baker, J. R. Brainard, D. L. Alumbaugh, and D. LaBrecque (2002), A geostatistically based inverse model for electrical resistivity surveys and its applications to vadose zone hydrology, Water Resour. Res., 38(12), 1278, doi:10.1029/2001WR001204.

A. Binley, Department of Environmental Science, Lancaster University, Lancaster LA1 4YW, UK.

N. Linde, Institute of Geophysics, ETH Hönggerberg, CH-8093 Zurich, Switzerland. (linde@aug.ig.erdw.ethz.ch)

L. B. Pedersen and A. Tryggvason, Department of Earth Sciences/ Geophysics, Uppsala University, SE-75236 Uppsala, Sweden.

A. Revil, Department of Hydrogeophysics and Porous Media, CNRSCEREGE, Université Paul Cézanne, BP 80, F-13545Aix-en-Provence Cedex 04, France. 